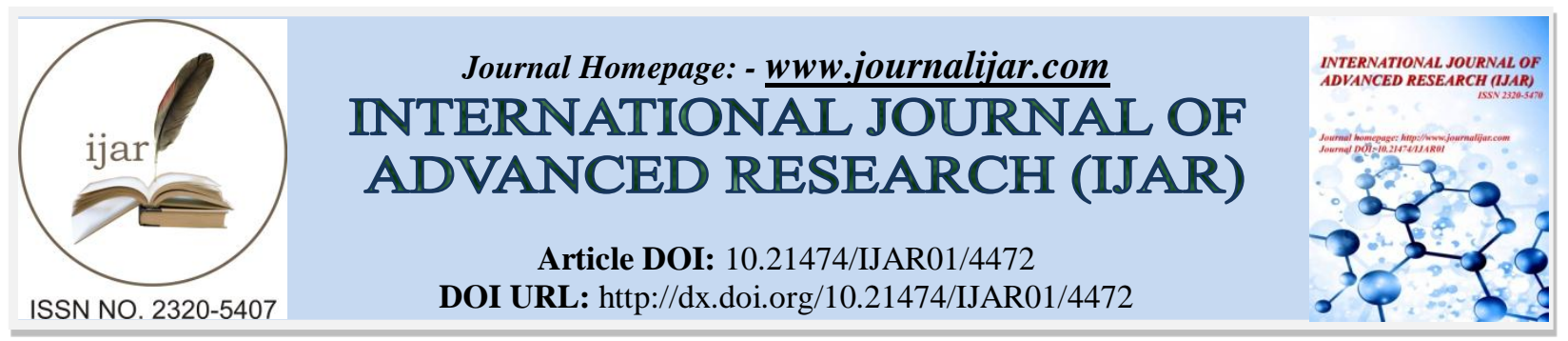

RESEARCH ARTICLE

\title{
DIVERSITY AND DISTRIBUTION OF ENDEMIC ANGIOSPERMS IN GUJARAT.
}

Karan Gopalbhai Rana ${ }^{1}$ and Padamnabhi Shanker Nagar ${ }^{2}$.

1. PhD student, Department of Botany, Faculty of Science, The Maharaja Sayajirao University of Baroda, Vadodara - 390002, Gujarat, India.

2. Assistant Professor, Department of Botany, Faculty of Science, The Maharaja Sayajirao University of Baroda, Vadodara - 390002, Gujarat, India.

\section{Manuscript Info}

\section{Manuscript History}

Received: 13 April 2017

Final Accepted: 15 May 2017

Published: June 2017

Key words:-

Gujarat, endemics, angiosperms, diversity

\section{Abstract}

Endemic plants represent a small but important part of the flora of Gujarat; still they have not been assessed for their status till date. The present estimate is first of its kind that encompasses all Indian endemics found in Gujarat state that is based on literature review and field surveys. The present findings revealed documentation of 192 Indian endemics, with dicotyledons dominating the endemic flora with 137 taxa $(71 \%)$ belonging to 97 genera under 33 families, whereas monocotyledons represented by 55 taxa (29\%) belonging to 37 genera under 9 families. There are seven species which are exclusively endemic to Gujarat state that are Helichrysum cutchicum, Ischaemum sayajiraoi, Rorippa cochlearioides, Solanum purpureilineatum, Spodiopogon aristatus, Tamarix kutchensis and Tephrosia jamnagarensis. Top five families contribute $57 \%$ of the total Indian endemics reported from Gujarat; Fabaceae has the largest number (31), which is followed by Poaceae (27), Acanthaceae (20), Asteraceae (12) and Orchidaceae (10). An analysis of the life-form types shows that endemic herbs $(66 \%)$ dominate the study area, followed by shrubs $(12 \%)$, trees $(11 \%)$, climbers $(6 \%)$, undershrubs (3\%) and climbing shrubs (2\%). Analysis on the district-wise distribution of Indian endemics shows that the Dangs has maximum number of Indian endemics with 115 taxa, followed by Valsad (104), Narmada (71), Junagadh (63) and Panchmahal (57). In conclusion, the present study demonstrates that endemics are mostly concerted in southern Gujarat region; they are primarily herbaceous; while some of them are known with just a few additional collections.

Copy Right, IJAR, 2017,. All rights reserved.

\section{Introduction:-}

Endemism denotes confinement of a taxon to a restricted area which may range from a small habitat to a biogeographical region, usually isolated by geographical barriers. Studies on plant endemism provide an insight into the centres of diversity and highlight the indigenous nature of its biological diversity. Endemism is a significant factor in diversity assessment and conservation. Reduction and fragmentation of wild population and habitats are occurring at an accelerating scale. These have resulted in small isolated populations (endemic plants) that are at risk 
of extinction for an increasing number of taxa. For such populations, more intensive management becomes necessary for their survival. Hence, in conservation efforts priority should be always given for endemics. So it becomes very essential that threatened taxa should be repeatedly searched for in their type localities for correct assessment of their status and proper management and conservation.

Gujarat state has about $8.7 \%$ of its total geographical area under four national parks and 23 wildlife sanctuaries which is more than double the national average and $13.8 \%$ area under wetlands which is about one third of the national area under wetland. Because of its unique geographical location, divergent climatic conditions, flat and undulating topography, vast expanse of the unique and fascinating deserts of Rann of Kachchh, the mighty ocean with a long coastline, vast grasslands, extensive thorny and dry deciduous forests in Saurasthra and northern Gujarat; moist to semi-moist forests in southern Gujarat and large area under a variety of wetlands and three hill ranges, namely, Aravalis, Vindhyas and Western Ghats, Gujarat has led to harbor a unique \& rich biodiversity in the state. Recent findings by Jani (2014) reveals that the angiosperm flora of Gujarat comprises of 2555 taxa (2320 species, 60 subspecies, 169 varieties and 6 forma), which represents nearly $16.8 \%$ of the national flora in its varied phytogeographical regions such as desert, semi-arid scrublands, coastal-reef-rich mangrove belts and dry-moist deciduous forests interspersed with fertile agricultural fields.

Endemic and threatened plants represent a small but important part of the flora of Gujarat. They are very susceptible to the effects of environmental change, because they occur in small populations or at scattered localities and are often confined to unique and localized habitats. The causes of such environmental change are biotic activities such as land clearing, cutting, lopping, overgrazing, the development of man-made forests (monoculture) and industrial pollution. The result of all such activities will be the dwindling of number of certain habitat-specific, wild plants because of change in their habitats. It is therefore urgently required to make an inventory of all such endemic and threatened plant species and to initiate measures to conserve the specific habitats and consequently the species occupying such habitats. The purpose of this list is to provide all relevant information on such plants of Gujarat.

Secondly, over the past years, the forest department has developed many man-made forests (mostly monocultures) in the area for different purposes like providing shelter belts, green belts and for firewood. This also has resulted in the loss of few wild habitats. Although few areas have been partially protected, many others are urgently in need of full-scale protection. Strict laws should be passed and enforced for the preservation of rare species and their habitats and strict penalties should be imposed on defaultors. Until such measures are taken up, it is proposed that the endemic and threatened plants should be brought under cultivation in central nurseries, botanical gardens, etc. and later reintroduced into their wild habitats. Then only there will be grounds for optimism for the protection of endemic and threatened plants and their habitats in Gujarat.

Studies on endemism and their conservation have received petite attention in Gujarat, and except for a few publications, there is little information on this subject. From the available literature it was determined that information on endemics in Gujarat was altogether lacking. An acute need was therefore felt to assess the present situation, locate important lacunae and stimulate studies on as large a scale as possible on threatened plants.

\section{Study Area:-}

The study site, Gujarat state, is located on the west coast of India and lies between $20^{\circ} 02^{\prime}$ and $24^{\circ} 41^{\prime}$ North latitudes, and $68^{\circ} 08^{\prime}$ and $74^{\circ} 23^{\prime}$ East longitudes covering a land area of $196,024 \mathrm{sq} . \mathrm{km}$.

Gujarat comprises 33 districts, of which Kachchh is the largest holding 23 percent, while Dangs is the smallest holding less than one percent of its total geographical area. The state is broadly classified into Kachchh, Saurashtra, Northern, Central and Southern regions (Fig. 1). 


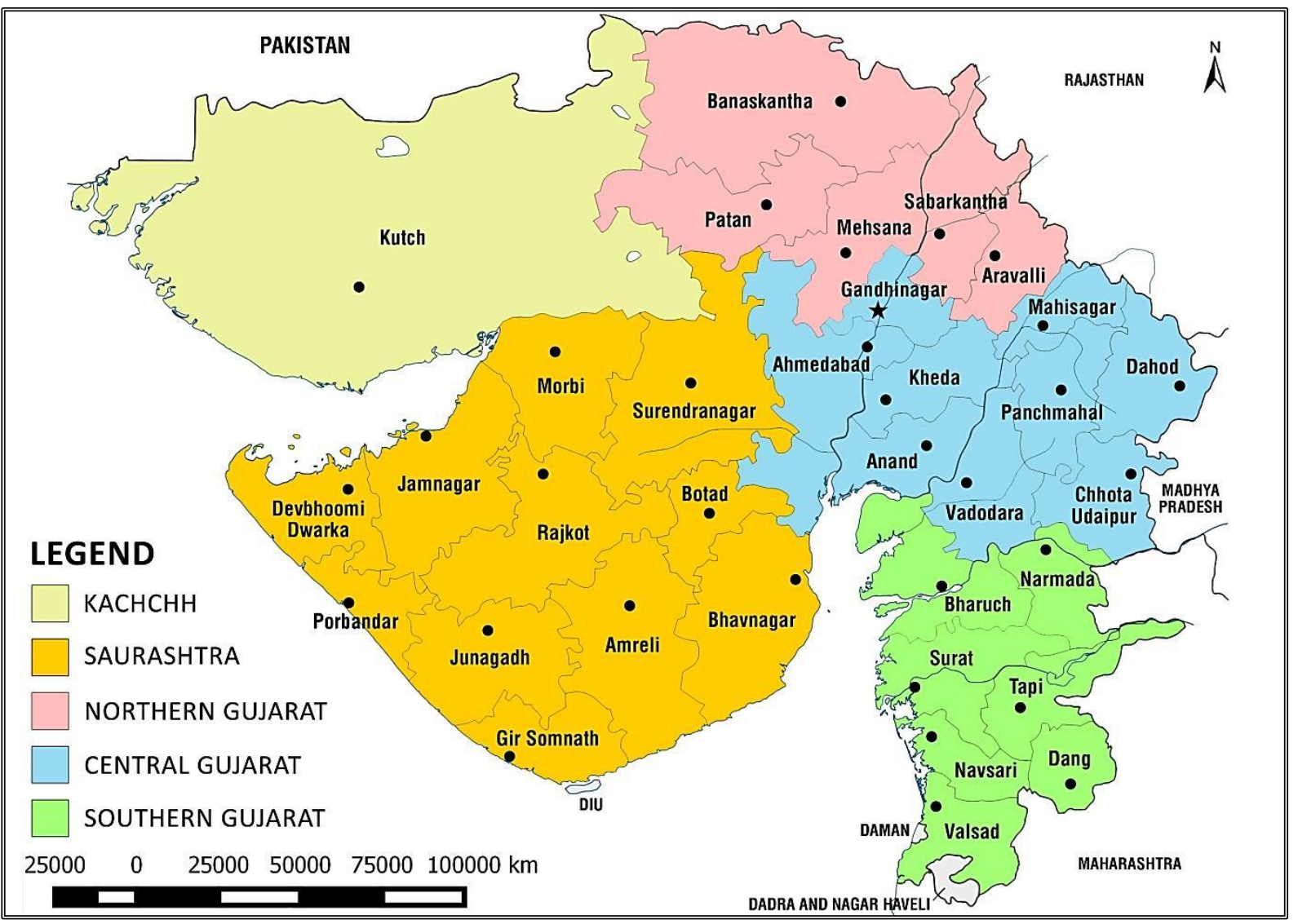

Figure 1:- Study Area.

\section{Kachchh:-}

The North-west arid region encompasses the Kachchh district. This region receives very little annual rainfall between 300 and $400 \mathrm{~mm}$, rendering it totally arid. The total geographic area of the region is 40,890 sq. $\mathrm{km}$. Since sandy and saline soils are not supportive of agricultural activities the region has the lowest geographical area under agriculture. The Kachchh region consists of the Ranns, which are salt-encrusted wastelands and rises only a few meters above sea level. Inundated during the monsoons they are divided into the great Rann to the north and the little Rann of Kutch to the east, the Banni Plains between the great Rann and the rocky mainland and the hilly region with the island belt of four rocky projections rising above the Rann, the Kachchh mainland, and the southern coastal plains.

\section{Saurashtra:-}

The Saurashtra region includes the districts of Amreli, Bhavnagar, Rajkot, Bhavnagar, Jamnagar, Surendranagar, Junagadh, and Porbandar. The total geographic area of the region consists of 60,950 sq. km. The climate here is dry sub-humid with very low average rainfall at 500 to $800 \mathrm{~mm}$ annually. The soil here is predominantly shallow to medium black and calcareous.

\section{Northern Gujarat:-}

The North Gujarat region includes the districts of Sabarkantha, Mehsana, Banaskantha, Aravalli and Patan. Its total geographic area comprises $28,910 \mathrm{sq}$. $\mathrm{km}$. This region has a very small area under forests. It receives 500 to $800 \mathrm{~mm}$ of annual average rainfall and the climate varies from arid to semi-arid. Grey Brown loamy, alluvial soils are predominant in this region.

\section{Central Gujarat:-}

The Central Gujarat region includes the districts of Kheda, Anand, Vadodara, Ahmedabad, Gandhinagar, Mahisagar, Chhota Udepur, Panchmahals and Dahod. The total geographic area of the region comprises $34,130 \mathrm{sq} . \mathrm{km}$. The 
forested area is not extensive in the region and this region leads in agricultural development. Annual rainfall averages from 800 to $1000 \mathrm{~mm}$ and the climate is semi-arid while the soil is medium black in nature.

\section{Southern Gujarat:-}

The southern region includes the districts of Surat, Bharuch, Valsad, Dangs, Tapi, Narmada and Navsari. Its total geographic area is $23,220 \mathrm{sq} . \mathrm{km}$. The region has the highest forested area in the state. Annual rainfall averages between 1,000 and $1,500 \mathrm{~mm}$ and the climate varies from semi-arid to dry sub humid. Deep black and coastal alluvial soil is predominant in this region.

Gujarat state is endowed with great diversity of flora distributed over $19,178 \mathrm{sq} . \mathrm{km}$, of its forest area, nearly $9.78 \%$ to the total geographical area. $8.78 \%$ of its total geographical area is covered by four national parks and 21 sanctuaries endowed with rich flora.

\section{Method:-}

The present estimate is first of its kind that encompasses all vascular plants of the state and is based on extensive survey of literature.

A list of endemic plants from the study area was prepared using $\mathrm{PhD}$ thesis:

Oza, G.M. (1961); Sabnis, S.D. (1967); Bedi, S.J. (1968); Deshpande, M.B. (1968); Suryanarayana, B. (1968); Yogi, D.V. (1970); Bhatt, R.P. (1971); Patel R.M. (1971); More, P.G. (1972); Karetala, Y.Y. (1973); Padate, S.N. (1973); Vyas, K.J. (1973); Parabia M.H. (1974); Thaker, D.N. (1974); Bhatt, R.G. (1975); Desai, M.J. (1976); Sanjappa, M. (1977); Menon, A.R.R. (1979); Yadav, S.R. (1979); Bhagwanani P.P. (1980); Joshi, J.V. (1980); Vora, H.M. (1980); Rao, K.S.S. (1981); Gopal, G.V. (1983); Joshi, M.C. (1983); Reddy, A.S. (1987); Thakrar, N.K. (1987); Oza, A.R. (1991); Bhatt, J.B. (1993); Binojkumar, M.S. (1993); Pradeepkumar, G. (1993); Raole, V.M. (1993); Joshi, K.I. (1994); Anjaria, K.B. (2002); Desai, B.S. (2002); Rao, V.R. (2002); Baxi, U.S. (2003); Sisodia, P.S. (2007); Patel, M.K. (2009); Sharma, A.K. (2010); Desai, R.J. (2012); Rao, V.H. (2012); Patel, S.L. (2013); Tadvi, D.S. (2013); Jani, M.D. (2014); Bhambra, G.P. (2015), and from available floristic works:

Thaker, J.I. (1910); Saxton, W.T. and Sedgwick, L.G. (1918); Santapau, H. (1953); Santapau, H. and Raizada, M.B. (1954); Phatak, V.G. and Joshi, B.B. (1955); Chavan, A.R. and Sabnis, S.D. (1958); Cooke, T. (1958); Chavan, A.R. and Mehta, A.R. (1958a); Chavan, A.R. and Mehta, A.R. (1958b); Bharati S.G. (1959); Phatak, V.G. and Oza, G.M. (1959); Chavan, A.R. and Oza, G.M. (1960); Chavan, A.R. and Sabnis, S.D. (1960); Chavan, A.R. and Oza, G.M. (1962); Sabnis, S.D. (1962); Santapau, H. (1962); Sanjappa, M. (1992); Chavan, A.R., Bedi S.J. and Sabnis, S.D. (1963); Patel, R.I. (1965); Chavan, A.R. and Oza, G.M. (1966); Santapau, H. and Kapadia, Z. (1966); Shah, G.L. (1967); Bedi, S.J., Sabnis, S.D. and Bhatt, R.P. (1968); Inamdar, J.A. (1968); Rao, R.S. and Deshpande, U.R. (1968); Shah G.L. and Suryanarayana B. (1968); Padate, S.N. (1969); Inamdar, J.A. and Patel, R.M. (1971); Patel, R.I. (1971); Sabnis, S.D. and Bedi S.J. (1971); Shah G.L. and Patel A.I. (1971); Bhatt R.P. and Sabnis S.D. (1972); Malhotra, S.K. and Wadhwa, B.M. (1973); Shah G.L. and Yogi D.V. (1974); Bahadur K.N. and Bennet S.S.R. (1978); Shah, G.L. (1978); Raghavan, R.S., Wadhwa, B.M., Ansari, M.Y. and Rao, R.S. (1981); Patil, S.N. and Sabnis, S.D. (1982); Shah, G.L. (1983); Bole, P.V. and Pathak, J.M. (1988); Mathew, T. and Nayar, M.P. (1989); Shetty, B.V. and Pandey, R.P. (1992); Nagar P.S., Sata S.J., and Pathak S.J. (2003); Parmar, P.J. and Singh, V. (2003); Nagar, P.S. (2005); Nagar, P.S. (2007); Nagar, P.S. (2008); Pandey R.P., Singh V. and Parmar P.J. (2009); Rajendrakumar, S. and Kalavathy, S. (2010); Raole V.M., Desai R.J. and Veldkamp, J.F. (2011); Patel Y.S., Patel R.M., Joshi P.N. and Dabgar Y.B. (2011); Singhadiya, M.K., Padhye, P.M. and Pandey, R.P. (2011); Joshi, P.N., Joshi, E.B. and Jain B.K. (2012); Meena, S.L. (2012); Parmar, P.J. (2012); Joshi, P.N., H.B. Soni, Sunderraj S.F.W. and Joshua J. (2013); Bhatt, M.R. and Nagar, P.S. (2014); Meena, S.L. (2014a); Meena, S.L. (2014b); Patel R., Patel Y., Kumar A., Mahato R. and Kumar V. (2014); Meena, S.L. and Krishna P.H. (2015); Punjani, B.L., Desai, P. and Patel, S.K. (2015); Satish K.V., Pasha S.V., Hari Krishna P. and Reddy C.S. (2015); Singh P., Karthigeyan K., Lakshminarasimhan P. and Dash S. S. (2015).

Preliminary field observations were carried out in the study area for endemics. A computerized database was generated incorporating details on their distribution, life-form type, habitat, phenology and local names.

Consulting the published literature, unpublished research reports and $\mathrm{PhD}$ dissertations pertained to the flora of Gujarat; a comprehensive checklist of angiosperms of Gujarat has been prepared. A few field surveys were organized in various geographic zones of Gujarat in order to enumerate some selected species. Plants were identified 
using regional, national and international Floras. This checklist is further processed through the following steps in order to construct taxonomically rich and accurate database.

- Each and every scientific name is looked for the correct orthography from the reliable International Plant Names Index (www.ipni.org) database and corrected wherever required.

- Scientific names are checked for their validity and legitimacy. Also, basionyms and synonyms are provided.

- Distribution data is updated based on the information gathered from the published literature.

- Field surveys were organized in various geographic zones of Gujarat in order to get field data. During these surveys various species were collected, processed and identified using relevant floras.

- The Botanical Survey of India, Arid Zone Circle, Jodhpur; the Botanical Survey of India, Western Regional Center, Pune; Sardar Patel University, Vallabh Vidyanagar; Veer Narmad South Gujarat University, Surat; Government Science College, Gandhinagar; GUIDE, Kachchh; Sahjeevan, Bhuj; Junagadh Agriculture University, Junagadh; Anand Agricultural Univserity, Anand; B.K.M. science college, Valsad, and Waghai Botanical Garden, Dangs were visited for consulting relevant literature so as to update the distribution data of the enumerated species.

\section{Results:-}

The survey resulted in the documentation of 192 Indian endemic taxa found in Gujarat state. Recently, Singh et al. (2015) enumerated a total of 4303 flowering plant species and infraspecfic taxa recorded as strict endemics to the Indian political boundary. Among them, dicotyledons dominate the endemic flora with 3170 taxa (72\%) belonging to 723 genera under 127 families, whereas monocots are represented by 1133 taxa (26\%) belonging to 254 genera under 27 families. As per the records, Gujarat ranks $25^{\text {th }}$ having six species exclusively endemic to the state i.e., Ischaemum sayajiraoi, Spodiopogon aristatus, Tephrosia jamnagarensis, Tamarix kutchensis, Helichrysum cutchicum and Pycreus dwarkensis. But Pycreus dwarkensis has been reported in Pakistan (eFloras, 2017), so it is no more an endemic. But, Rorippa cochlearioides and Solanum purpureilineatum are also exclusively endemic to Gujarat. So as per the present analysis, there are total seven species exclusively endemic to Gujarat state.

In the endemic vascular plants of India database (Singh et al., 2015) only 55 endemic taxa have reported Gujarat as a locality, but the present study added 137 more such Indian endemics being reported from Gujarat state which are marked with an asterisk (*).

\section{Araceae Juss:-}

*Amorphophallus commutatus (Schott) Engl.

Herb; Fl. and Fr.: June - August

Dahod Dt.: Ratanmahal (Bedi, 1968); Dang Dt.: Galkund (Yadav, 1979), Ahwa, Malegaon, Pipaldahad, Subir (Suryanarayana, 1968); Gir somnath Dt. (Sisodia, 2007); Junagadh Dt.: Chorwad (Bole and Pathak, 1988); Narmada Dt. (Patel, 1971): Mathavali, Sagai, Waghumar (Pradeepkumar, 1993); Panchmahal Dt.: Pavagadh (Oza, 1961); Surat Dt.: Umarpada (Yadav, 1979); Valsad Dt. (Inamdar and Patel, 1971): Pindval (Reddy, 1987), Kaprada (Rao, 2012), Parnera (Yadav, 1979), Dharampur, Nana Pondha (Vora, 1980), Pardi (More, 1972)

*Arisaema murrayi (J.Graham) Hook.

Herb; Fl. and Fr.: June - August

Dahod Dt.: Kanjeta (Bedi, 1968); Dang Dt. (Tadvi, 2013): Ahwa, Don, Malegaon, Saputara; Valsad Dt. (Vora, 1980): Dahikhed (Rao, 2012)

Amaryllidaceae J. St.-Hil:*Crinum brachynema Herb. Herb; Fl. and Fr.: July - August

Valsad Dt. (Inamdar and Patel, 1971): Dharampur (Inamdar, 1968; Vora, 1980), Kaprada (Rao, 2012)

*Pancratium parvum Dalzell

Herb; Fl. and Fr.: June - July

Bhavnagar Dt. (Oza, 1991); Valsad Dt. (Inamdar and Patel, 1971)

Asparagaceae Juss:-

*Chlorophytum borivilianum Santapau and R.R.Fern.

Herb; Fl. and Fr.: June - July 
Banaskantha Dt.: Danta (Meena, 2012), Jessore wildlife sanctuary; Dang Dt. (Shah, 1978; Tadvi, 2013): Ahwa (Shah and Suryanarayana, 1967), Malegaon (Suryanarayana, 1968), Bardipada, Kalibel, Mahal, Subir, Waghai (Rao, 2002), Ghoghli, Pimpri, Shivghat; Narmada Dt. (Sharma, 2010): Dediapada, Sagai; Sabarkantha Dt.: Vijaynagar; Surat Dt.

*Chlorophytum malabaricum Baker

Herb; Fl. and Fr.: June

Dang Dt. (Shah, 1978; Tadvi, 2013): Ahwa (Suryanarayana, 1968)

Hyacinthaceae:-

Dipcadi ursulae var. longiracemosum Deb and S.Dasgupta

Herb; Fl. and Fr.: August

Jamnagar Dt.: Rozi (Bole and Pathak, 1988); Junagadh Dt.: Girnar hill (Shah, 1983)

Orchidaceae Juss:-

*Aerides maculosa Lindl.

Herb; Fl. and Fr.: July - January

Dang Dt. (Tadvi, 2013; Nagar and Bhatt, 2015): Ahwa, Malegaon, Subir (Suryanarayana, 1968); Junagadh Dt.: Sasan-Gir; Navsari Dt.: Bansda; Panchmahal Dt. (Nagar and Bhatt, 2015): Pavagadh (Oza, 1961); Valsad Dt. (Nagar and Bhatt, 2015): Dhamni, Pangarbari (Reddy, 1987), Kaprada (Rao, 2012)

*Dendrobium barbatulum Lindl.

Herb; Fl. and Fr.: March - May

Dang Dt. (Tadvi, 2013): Ahwa, Malegaon (Suryanarayana, 1968), Don (Nagar and Bhatt, 2015); Narmada Dt. (Nagar and Bhatt, 2015): Mohbi (Pradeepkumar, 1993); Panchmahal Dt. (Nagar and Bhatt, 2015); Valsad Dt. (Nagar and Bhatt, 2015): Girnara, Pangarbari (Reddy, 1987), Morai, Varoli Talat (Rao, 2012)

*Dendrobium microbulbon A.Rich.

Herb; Fl. and Fr.: December - May

Dang Dt. (Tadvi, 2013; Nagar and Bhatt, 2015): Ahwa, Malegaon (Suryanarayana, 1968), Bardipada, Dungerda, Galkund, Kalibel, Kasa, Mahal, Subir (Rao, 2002); Narmada Dt.: Kokam, Mathavali, Sagai, Waghumar (Pradeepkumar, 1993), Dediapada, Fulsar, Piplod (Rao, 2002), Ninai; Navsari Dt.: Khambhala, Tadpada (Desai, 1976), Bansda; Surat Dt.: Zankhri; Valsad Dt.: Kaprada

*Dendrobium ovatum (L.) Kraenzl. [= Epidendrum ovatum L.]

Herb; Fl. and Fr.: February - March

Dang Dt. (Tadvi, 2013): Subir (Suryanarayana, 1968), Ganja, Kansa; Valsad Dt.: Kaprada

*Eulophia ochreata Lindl.

Herb; Fl. and Fr.: June - October

Dang Dt.: Galkund (Rao, 2002), Ahwa (Nagar and Bhatt, 2015), Mahal; Narmada Dt.: Kelda; Panchmahal Dt.: Khodwa (Santapau and Kapadia, 1966)

*Habenaria foliosa A.Rich. [= Habenaria foliosa A.Rich. var. gibsonii (Hook.f.) Bennet; Habenaria gibsonii Hook.f. var. foetida Blatt. and McCann; Habenaria gibsonii Hook.f. var. gibsonii; Habenaria gibsonii Hook.f. var. foliosa (A. Rich.) Santapau and Kapadia]

Herb; Fl. and Fr.: July - September

Chhota udepur Dt.; Dang Dt. (Tadvi, 2013; Nagar and Bhatt, 2015): Malegaon (Suryanarayana, 1968); Junagadh Dt.: Datar (Bhatt \& Nagar); Narmada Dt.: Namgir (Pradeepkumar, 1993), Ghatoli; Navsari Dt.: Kanai khadi, Mankunia, Vati, Vedchha; Valsad Dt. (Nagar and Bhatt, 2015): Dungri, Ghadoi

*Habenaria grandifloriformis Blatt. and McCann

Herb; Fl. and Fr.: July - August

Dang Dt. (Tadvi, 2013; Nagar and Bhatt, 2015): Malegaon, Saputara (Suryanarayana, 1968); Valsad Dt. (Nagar and Bhatt, 2015): Pindval, Sutharpada (Reddy, 1987), Vavar (Rao, 2012)

*Habenaria longicorniculata J.Graham

Herb; Fl. and Fr.: August - September

Chhota udepur Dt.; Dang Dt. (Tadvi, 2013): Malegaon (Suryanarayana, 1968), Chinchli, Saputara; Valsad Dt.: Sanjan

*Oberonia brunoniana Wight [= Malaxis brunoniana (Wight) Rchb. f.]

Herb; Fl. and Fr.: February - March

Dang Dt.: Kansa

*Peristylus stocksii (Hook.f.) Kraenzl. [= Habenaria stocksii Hook.f.] 
Herb; Fl. and Fr.: July - September Dang Dt. (Tadvi, 2013): Waghai; Narmada Dt.: Mohbi (Pradeepkumar, 1993); Panchmahal Dt.; Valsad Dt. (Nagar and Bhatt, 2015)

Cyperaceae Juss:-

*Fimbristylis lawiana (Boeckeler) J.Kern [= Scirpus lawianus Boeckeler]

Herb; Fl. and Fr.: August - October

Anand Dt.: Umeta; Dang Dt.: Ahwa, Malegaon, Saputara (Suryanarayana, 1968; Parabia, 1974); Vadodara Dt.: Nimeta (Parabia, 1974); Valsad Dt.: Kaprada (Reddy, 1987), Malghar (Rao, 2012)

*Fimbristylis woodrowii C.B. Clarke

Herb; Fl. and Fr.: August - October

Bharuch Dt.: River Dhadhar at Shahpura; Dang Dt.: Ahwa (Suryanarayana, 1968), Waghai (Parabia, 1974); Narmada Dt. (Patel, 1971); Panchmahal Dt.: Tuwa (Deshpande, 1968; Parabia, 1974); Surat Dt.: Dumas (Joshi, 1980); Vadodara Dt. (Parabia, 1974): riverbank of Dhadhar at Shahapura (Sabnis, 1962); Valsad Dt. (Inamdar and Patel, 1971): Pangarbari (Reddy, 1987), Panas (Rao, 2012), Dharampur, Kaprada, Nana Pondha (Vora, 1980)

*Fuirena tuwensis M.B. Deshp. and G.L. Shah

Herb; Fl. and Fr.: October - November

Dadra Nagar Haveli (Sabnis and Bedi, 1971); Panchmahal Dt.: Bakrol, Navagam, Tuwa, Veganpura (Deshpande, 1968; Parabia, 1974)

*Pycreus malabaricus C.B. Clarke

Herb; Fl. and Fr.: August - November

Dang Dt. (Tadvi, 2013): Ahwa (Suryanarayana, 1968; Parabia, 1974); Navsari Dt.: Maroli; Porbandar Dt.: Bileshwar (Menon, 1979); Tapi Dt.: Hindla, Songadh; Valsad Dt.: Pindval (Reddy, 1987), Kaprada (Rao, 2012), Manda

*Scleria stocksiana Boeckeler

Herb; Fl. and Fr.: August - September

Sabarkantha Dt. (Sabnis, 1962): Talod (Saxton and Sedgwick, 1918); Vadodara Dt.: Nimetta (Chavan and Sabnis, 1958)

Eriocaulaceae Martinov:-

*Eriocaulon cuspidatum Dalzell

Herb; Fl. and Fr.: September - November

Chhota udepur Dt.: Kavant (Thaker, 1974)

*Eriocaulon elenorae Fyson

Herb; Fl. and Fr.: September - October

Mehsana Dt. (Shah and Yogi, 1974); Narmada Dt. (Patel, 1971): Kokam (Pradeepkumar, 1993), Vankol (Yadav, 1979); Panchmahal Dt.: Tuwa (Deshpande, 1968); Sabarkantha Dt.: Idar; Valsad Dt. (Inamdar and Patel, 1971): Dharampur (Reddy, 1987), Kaprada (Rao, 2012), Nana Pondha (Vora, 1980)

\section{Poaceae Barnhart:-}

*Arthraxon inermis Hook.f.

Herb; Fl. and Fr.: August - November

South Gujarat (Patel, 1965); Dang Dt. (Desai, 2012; Tadvi, 2013); Junagadh Dt.: Petwad (Menon, 1979); Narmada Dt.: Dabka, Namgir, Sagai (Pradeepkumar, 1993); Navsari Dt. (Desai, 2012); Valsad Dt. (Desai, 2012)

*Arthraxon meeboldii Stapf

Herb; Fl. and Fr.: August - October

South Gujarat (Patel, 1965); Dang Dt.; Valsad Dt.

*Arundinella ciliata (Roxb.) Nees ex Miq.

Herb; Fl. and Fr.: August - October

Dang Dt. (Desai, 2012; Tadvi, 2013); Navsari Dt. (Desai, 2012); Valsad Dt. (Desai, 2012): Kaprada, Lavkar, Mandva, Nana Pondha

*Arundinella metzii Hochst. ex Miq.

Herb; Fl. and Fr.: September - November

South Gujarat, on nalla-banks (Patel, 1965); Banaskantha Dt.: Dantiwada, Palanpur (Patel, 2009); Dang Dt.: Saputara (Yadav, 1979); Narmada Dt.: Kanjal, Sagai, Mohbi, Waghumar (Pradeepkumar, 1993); Valsad Dt. (Vora, 1980): Tutarkhed (Reddy, 1987), Kaprada (Rao, 2012) 
*Arundinella tuberculata Munro ex Lisboa

Herb; Fl. and Fr.: August

Rajkot Dt.: Valadhari (Bole and Pathak, 1988)

*Capillipedium filiculme (Hook.f.) Stapf [= Andropogon filiculmis Hook.f.]

Herb; Fl. and Fr.: August - September

Gujarat (Patel, 1965); Bhavnagar Dt.: Palitana (Menon, 1979); Dang Dt.: Galkund (Yadav, 1979); Jamnagar Dt. (Bole and Pathak, 1988); Panchmahal Dt.: Pavagadh (Chavan and Mehta, 1958b; Oza, 1961); Porbandar Dt.: Barda hill (Bole and Pathak, 1988); Surat Dt. (Desai, 2012); Tapi Dt. (Desai, 2012); Vadodara Dt.: Shardadungri (Padate, 1973), Savli (Padate, 1969); Valsad Dt.: Pendha (Yadav, 1979), Parnera (More, 1972)

*Capillipedium huegelii (Hack.) A. Camus [= Andropogon huegelii Hack.]

Herb; Fl. and Fr.: August - September

South Gujarat (Patel, 1965); Amreli Dt. (Menon, 1979); Dahod Dt.: Bendol, Ratanmahal temple (Bedi, 1968); Narmada Dt.: Kalvat, Kelda, Ninaighat (Pradeepkumar, 1993); Rajkot Dt.: Hingolgadh (Menon, 1979); Sabarkantha Dt.: Khedbrahma (Bhatt, 1971; Bhatt and Sabnis, 1972); Valsad Dt.: Pendha (Yadav, 1979)

*Coelorachis clarkei (Hack.) Blatt. and McCann [= Rottboellia clarkei Hack.; Mnesithea clarkei (Hack.) de Koning and Sosef; Manisuris clarkei (Hack.) Bor ex Santapau]

Herb; Fl. and Fr.: September - October

Narmada Dt.: Khamar, Kokam, Sagai (Pradeepkumar, 1993); Valsad Dt.: Kaprada (Desai, 2012)

*Cymbopogon gidarba (Buch.-Ham. ex Steud.) A. Camus [= Andropogon gidarba Steud.]

Herb; Fl. and Fr.: December - March

Bharuch Dt.; Junagadh Dt.: Girnar (Menon, 1979); Mehsana Dt.: Kadi (Yogi, 1970); Narmada Dt.: Rajpipla; Porbandar Dt.: Bileshwar (Menon, 1979); Sabarkantha Dt.: Khedbrahma (Yogi, 1970; Bhatt, 1971; Bhatt and Sabnis, 1972), Mahudi, Pahada (Shah and Yogi, 1974; Yogi, 1970)

*Cynodon barberi Rang. and Tadul.

Herb; Fl. and Fr.: September

Sabarkantha Dt.: Himmatnagar; Surat Dt.: Vadpada (Desai, 2012)

*Dimeria stapfiana C.E. Hubb. and Pilg.

Herb; Fl. and Fr.: September - November

Valsad Dt.: Dharampur, Kaprada (Desai, 2012)

*Glyphochloa forficulata (C.E.C. Fisch.) Clayton [= Manisuris forficulata C.E.C.Fisch.]

Herb; Fl. and Fr.: September - December

Dang Dt. (Tadvi, 2013): Gadvihir (Desai, 2012)

*Heteropogon ritchiei (Hook.f.) Blatt. and McCann [= Andropogon ritchiei Hook.f.]

Herb; Fl. and Fr.: October - December

Gujarat (Patel, 1965); Bhavnagar Dt.: Thadsar; Junagadh Dt.: Rukhmani Dungar, Tulsishyam (Bole and Pathak, 1988); Valsad Dt.: Kaprada (Desai, 2012)

*Isachne elegans Dalzell ex Hook.f.

Herb; Fl. and Fr.: October - December

Surat Dt.: Udhna-Magdalla road (Joshi, 1980)

*Ischaemum bombaiense Bor

Herb; Fl. and Fr.: September - December

Surat Dt.: Umarpada (Desai, 2012)

*Ischaemum diplopogon Hook.f.

Herb; Fl. and Fr.: September - October

Gujarat (Patel, 1965); Panchmahal Dt.: Pavagadh (Chavan and Mehta, 1958b); Dang Dt. (Desai, 2012; Tadvi, 2013)

*Ischaemum santapaui Bor

Herb; Fl. and Fr.: September - November

Dang Dt. (Tadvi, 2013): Girmal fall (Desai, 2012); Tapi Dt. (Desai, 2012); Valsad Dt.: Sanjan

Ischaemum sayajiraoi Raole and R.J. Desai

Herb; Fl. and Fr.: September - December

Dang Dt. (Desai, 2012; Tadvi, 2013); Surat Dt.: Umarpada (Desai, 2012); Tapi Dt. (Desai, 2012); Vadodara Dt.:

Bacrol, Bhayli (Raole et al., 2011)

*Iseilema anthephoroides Hack.

Herb; Fl. and Fr.: July - October 
Gujarat (Patel, 1965); Anand Dt.: Khambhat (Bhagwanani, 1980); Chhota udepur Dt.: Kavant (Thaker, 1974); Gandhinagar Dt.: Mansa (Meena, 2014a); Narmada Dt.: Rajpipla (Yadav, 1979); Panchmahal Dt.: Pavagadh (Chavan and Mehta, 1958b; Oza, 1961); Vadodara Dt. (Chavan and Mehta, 1958a): Harni (Sabnis, 1967)

*Lophopogon tridentatus (Roxb.) C.E.Hubb. [= Andropogon tridentatus Roxb.]

Herb; Fl. and Fr.: September - December

Gujarat (Patel, 1965); Panchmahal Dt.: Sulatpur (Chavan and Mehta, 1958b), Pavagadh (Oza, 1961)

*Parahyparrhenia bellariensis (Hack.) Clayton [= Andropogon bellariensis Hack.]

Herb; Fl. and Fr.: August - October

Rajkot Dt. (Thakrar, 1987)

Spodiopogon aristatus R.J. Desai and Raole

Herb; Fl. and Fr.: October - December

Dang Dt. (Desai, 2012; Tadvi, 2013); Navsari Dt. (Desai, 2012); Surat Dt. (Desai, 2012); Tapi Dt.: Bunadha (Desai and Raole, 2012); Valsad Dt.: Dabkhal (Desai and Raole, 2012)

Spodiopogon rhizophorus (Steud.) Pilg. [= Andropogon rhizophorus Steud.]

Herb; Fl. and Fr.: September - January

Saurashtra (Patel, 1965); Banaskantha Dt.: Jessore wildlife sanctuary, Balaram-Ambaji wildlife sanctuary (Meena, 2012); Chhota udepur Dt.: Kavant (Thaker, 1974); Dang Dt. (Desai, 2012; Tadvi, 2013): Galkund (Yadav, 1979); Devbhumi dwarka Dt.: Abhapara, Venu (Nagar, 2005); Junagadh Dt.: Junvaniya, Sasan (Bole and Pathak, 1988); Narmada Dt.: Rajpipla (Shah, 1967); Pachmahal Dt.: Pavagadh (Chavan and Mehta, 1958b; Oza, 1961); Sabarkantha Dt.: Khedbrahma (Bhatt, 1971; Bhatt and Sabnis, 1972); Tapi Dt. (Desai, 2012); Valsad Dt. (Desai, 2012): Sidhumbar, Tutarkhed (Reddy, 1987), Tiskari (Rao, 2012)

*Trilobachne cookei (Stapf) Schenck ex Henrard [= Polytoca cookei Stapf]

Herb; Fl. and Fr.: September - November

Saurashtra (Patel, 1965); Dang Dt. (Tadvi, 2013): Bhenskatri (Desai, 2012); Junagadh Dt.: Girnar foothill (Bole and Pathak, 1988); Valsad Dt.: Kapurnya, Moti Korval, Tamachhadi (Reddy, 1987), Tiskari (Rao, 2012)

*Triplopogon ramosissimus (Hack.) Bor [= Ischaemum ramosissimum Hack.]

Herb; Fl. and Fr.: October

Dang Dt. (Desai, 2012; Tadvi, 2013); Tapi Dt.: Tapti riverbank (Patel, 1965); Valsad Dt.: Kodba, Tamachhadi (Reddy, 1987), Kaprada (Rao, 2012)

*Tripogon jacquemontii Stapf

Herb; Fl. and Fr.: August - September

South Gujarat (Patel, 1965); Ahmedabad Dt.; Banaskantha Dt.: Amirgarh hills (Meena, 2012); Chhota udepur Dt.: Kavant (Thaker, 1974); Dang Dt. (Desai, 2012); Gandhinagar Dt.: Kalol (Meena, 2014a); Jamnagar Dt.: Jamvali (Bole and Pathak, 1988); Sabarkantha Dt.: Khedbrahma (Bhatt, 1971; Bhatt and Sabnis, 1972); Surat Dt. (Desai, 2012); Tapi Dt. (Desai, 2012)

*Tripogon lisboae Stapf

Herb; Fl. and Fr.: July - October

Chhota udepur Dt.: Kavant (Thaker, 1974); Dang Dt.; Narmada Dt.: Kevadiya

Zingiberaceae Martinov:-

*Curcuma decipiens Dalzell

Herb; Fl. and Fr.: June - December

Banaskantha Dt.: Hathidhara forest (Meena, 2012); Narmada Dt.: Fulsar, Sagai; Valsad Dt.: Kaprada

*Curcuma inodora Blatt. [= Curcuma purpurea Blatt.]

Herb; Fl. and Fr.: August - September

Banaskantha Dt.: Khaiwad, Danta (Meena, 2012); Chhota udepur Dt.: Ambadungar, Kadipani, Mithibor (Desai, 2002), Kavant (Thaker, 1974); Dahod Dt.: Ratanmahal (Bedi, 1968), Devgadh Baria; Dang Dt. (Tadvi, 2013): Ahwa, Malegaon, Subir (Suryanarayana, 1968); Gir somnath Dt. (Santapau and Raizada, 1954; Sisodia, 2007); Junagadh Dt.: Sapnes, Sasan (Menon, 1979), Girnar, Junvaniya (Bole and Pathak, 1988); Mehsana Dt. (Shah and Yogi, 1974); Narmada Dt.: Mathavali, Sagai, Shisha (Pradeepkumar, 1993); Panchmahal Dt.: Machi (Phatak and Oza, 1959), Pavagadh (Oza, 1961); Sabarkantha Dt.: Khedbrahma (Bhatt, 1971; Bhatt and Sabnis, 1972); Surat Dt.: Umarpada (Yadav, 1979); Valsad Dt. (Inamdar and Patel, 1971; Patel RM, 1971): Amba Talat, Moolgam (Reddy, 1987), Kaprada (Rao, 2012), Nana Pondha (Vora, 1980), Chival, Parnera, Udwada (More, 1972)

*Curcuma pseudomontana J. Graham

Herb; Fl. and Fr.: July - October

Dahod Dt.; Narmada Dt.; Panchmahal Dt.: Machi (Chavan and Oza, 1960; Oza, 1961) 
*Zingiber nimmonii (J. Graham) Dalzell [= Alpinia nimmonii J. Graham; Zingiber cernuum Dalzell]

Herb; Fl. and Fr.: January - February

Dang Dt. (Tadvi, 2013): Mahal (Suryanarayana, 1968)

\section{Ranunculaceae Juss:-}

Clematis hedysarifolia DC.

Climber; Fl. and Fr.: October - December

Dang Dt. (Tadvi, 2013): Shamgahan (Patel, 2013), Ahwa (MCJ157 Joshi, 1978), Borkhal, Sunda (Yadav, 1979), Saputara, Subir (Suryanarayana, 1968), Malegaon, Sagbara (Gopal, 1983); Narmada Dt.: Sagbara; Panchmahal Dt.: Vajpur (Patel, 2013); Tapi Dt.: Songadh, Vyara; Valsad Dt.: Pangarbari, Tamachhadi (Reddy, 1987; Patel, 2013), Penda (Yadav, 1979), Pindval, Vagval (Reddy, 1987), Varoli (Rao, 2012), Jogvel, Malanpada, Nana Pondha, Vahial (Vora, 1980), Rabada, Parnera (More, 1972)

*Clematis heynei M.A. Rau [= Clematis triloba B. Heyne ex Roth]

Climber; Fl. and Fr.: October - March

Dang Dt.: Saputara (Patel, 2013); Narmada Dt.; Navsari Dt.: Unai; Porbandar Dt.: Barda; Valsad Dt.: Dhamni (Patel, 2013), Varoli Talat (Rao, 2012)

Begoniaceae C. Agardh:-

*Begonia crenata Dryand.

Herb; Fl. and Fr.: September - October

Bhavnagar Dt. (Oza, 1991); Chhota udepur Dt. (Karetala, 1973): Mogra, Marchipani (Desai, 2002); Dang Dt. (Tadvi, 2013); Narmada Dt.: Ninaighat (Pradeepkumar, 1993)

\section{Cucurbitaceae Juss:-}

*Corallocarpus conocarpus (Dalzell and A.Gibson) Hook.f. [= Aechmandra conocarpa Dalzell and A.Gibson]

Climber; Fl. and Fr.: June - August

Ahmedabad Dt.: Dhandhuka (Patel et al., 2014); Arvalli Dt.: Dhansura (Patel, 2013); Bharuch Dt.: Malpore (Patel, 2013); Gir somnath Dt. (Sisodia, 2007); Jamnagar Dt.; Junagadh Dt.; Kachchh Dt. (Patel et al., 2011): Sural Pith, Bhuj, Wadasar, Tregdi (Joshi et al., 2013), Lala bustard sanctuary on Mandavi-Naliya road, Chamra, Bhujpar, Kharai village on Vayor road; Kheda Dt.: Malana Muvada, Ladvel (Patel, 2013); Sabarkantha Dt.: Netrawali (Parmar, 2012), Bayad, Merhan (Patel, 2013)

*Solena amplexicaulis (Lam.) Gandhi [= Bryonia amplexicaulis Lam.]

Climber; Fl. and Fr.: July - October

Chhota udepur Dt.; Dang Dt. (Tadvi, 2013): Shamgahan (Patel, 2013); Narmada Dt.: Dediapada (Patel, 2013), Mathavali, Ninaighat, Patvali (Pradeepkumar, 1993); Panchmahal Dt.: Pavagadh (Oza, 1961); Tapi Dt.: Vyara; Valsad Dt.: Dharampur, Pariya-Pardi, Navtad (Patel, 2013), Kaparada (Rao, 2012)

\section{Fabaceae Lindl:-}

*Alysicarpus hamosus Edgew. [= Alysicarpus procumbens (Roxb.) Schindl.; Hedysarum procumbens Roxb.] Herb; Fl. and Fr.: August - October

Anand Dt.: Khambhat (Bhagwanani, 1980); Banaskantha Dt.: Koteshwar (Meena, 2012); Bharuch Dt.: Gumandev; Bhavnagar Dt.: Palitana (Menon, 1979; Meena, 2014b); Chhota udepur Dt.: Kavant (Thaker, 1974); Dahod Dt.: Ratanmahal (Bedi, 1968), Devgadh baria; Dang Dt. (Tadvi, 2013): Ahwa, Subir (Suryanarayana, 1968); Devbhumi dwarka Dt.: Abhapar, Kileshwar, Ghumli, (Nagar, 2005); Gandhinagar Dt.: Halisa (Meena, 2014a), Mansa; Gir somnath Dt. (Santapau and Raizada, 1954; Sisodia, 2007); Junagadh Dt.: Sasan (Menon, 1979), Kansianes; Kachchh Dt.: Meva Rakhal (Bhatt, 1993), Guhar (Pandey et al., 2009), Ningal; Mehsana Dt.: Kadi; Narmada Dt.: Fulsar, Gichad, Namgir, Zadoli (Pradeepkumar, 1993), Gora, Gumandev, Kevadiya, Rajpipla (Patel, 1971); Navsari Dt.; Panchmahal Dt.: Pavagadh (Oza, 1961), Tuwa (Deshpande, 1968); Patan Dt.; Porbandar Dt.: Godhana, Satvirda, Adityana, Ranavav (Nagar, 2005); Sabarkantha Dt.: Khedbrahma (Bhatt, 1971), Samalaji, Bamaraji (Parmar, 2012), Idar, Prantij; Surat Dt. (Joshi, 1980): Pataldevi, Umarpada, Vankal (Yadav, 1979); Tapi Dt.; Vadodara Dt. (Sabnis, 1967): Savli (Padate, 1973); Valsad Dt.: Dhamni (Yadav, 1979), Avdha, Tutarkhed (Reddy, 1987), Umbergaon (Bhagwanani, 1980), Kaprada (Rao, 2012), Nana Pondha (Vora, 1980)

Alysicarpus pubescens J.S.Law

Herb; Fl. and Fr.: September - December

Amreli Dt.: Dhari (Gopal, 1983); Bharuch Dt.; Jamnagar Dt.: Ranjit Sagar (Santapau, 1962)

Bauhinia foveolata Dalzell [= Piliostigma foveolatum (Dalzell) Thoth.] 
Tree; Fl. and Fr.: October - March

Ahmedabad Dt. (Meena, 2014a); Dang Dt. (Shah, 1978; Tadvi, 2013): Ahwa (Yadav, 1979), Malegaon, Pipaldahad, Saputara (Suryanarayana, 1968), Bardipada, Don; Narmada Dt. (Sharma, 2010); Navsari Dt.: Mankunia, Raybor; Panchmahal Dt.; Surat Dt.: Divtan; Tapi Dt.: Hindla; Valsad Dt.: Fatepur, Ulaspedhi (Reddy, 1987), Mandva (Rao, 2012), Moti Korval (Rao, 2002), Kaprada, Nana Pondha (Vora, 1980), Chavshala

*Cajanus sericeus (Baker) Maesen [= Atylosia sericea Baker]

Shrub; Fl. and Fr.: August - December

Chhota udepur Dt.: Ambadungar (Thaker, 1974); Dang Dt. (Chavan and Oza, 1966); Valsad Dt.: Moti Korval, Pangarbari (Reddy, 1987)

Clitoria annua J.Graham [= Clitoria biflora Dalzell]

Herb; Fl. and Fr.: July - October

Ahmedabad Dt. (Meena, 2014a); Anand Dt.: Umeta; Banaskantha Dt.: Rinchhidiya (Meena, 2012); Chhota udepur Dt.: Dolariya (Desai, 2002), Kavant (Thaker, 1974; Desai, 2002); Dahod Dt.: Ratanmahal (Bedi, 1968); Dang Dt. (Tadvi, 2013): Ahwa, Malegaon, Subir (Suryanarayana, 1968); Devbhumi dwarka Dt.: Abhapar, Ghumli, (Nagar, 2005); Gir somnath Dt. (Sisodia, 2007); Junagadh Dt.: Sasan (Menon, 1979), Junvaniya (Santapau, 1962); Kachchh Dt. (Patel et al., 2011); Mehsana Dt. (Shah and Yogi, 1974); Narmada Dt.: Gumina, Mathavali, Sagai (Pradeepkumar, 1993), Kevadia, Gora, Jhagadiya (Patel, 1971); Panchmahal Dt.: Pavagadh (Oza, 1961); Porbandar Dt.: Godhana (Thaker, 1910); Sabarkantha Dt. (Parmar, 2012): Khedbrahma (Bhatt, 1971); Vadodara Dt.: Laxmi Vilas palace compound; Valsad Dt. (Patel RM, 1971): Ghotan (Rao, 2012), Kaprada, Nana Pondha (Vora, 1980), Hedri

*Crotalaria filipes Benth. [= Crotalaria filipes var. filipes]

Herb; Fl. and Fr.: October - February

Dang Dt. (Tadvi, 2013): Ahwa (Suryanarayana, 1968), Waghai (Gopal, 1983); Junagadh Dt.: Girnar (Santapau, 1962); Kachchh Dt. (Patel et al., 2011); Kheda Dt.: Utkantheshwar (Gopal, 1983); Narmada Dt.; Porbandar Dt. (Thaker, 1910); Valsad Dt. (Patel RM, 1971; Vora, 1980): Avdha, Kaprada (Rao, 2012)

*Crotalaria filipes var. trichophora (Baker) T.Cooke [= Crotalaria trichophora Benth. ex Baker f.]

Herb; Fl. and Fr.: October - March

Dang Dt. (Tadvi, 2013): Don; Gir somnath Dt. (Santapau and Raizada, 1954); Junagadh Dt.: Junvaniya (Santapau, 1962); Surat Dt.: Nana Varachha (Joshi, 1980); Valsad Dt. (Patel RM, 1971)

Crotalaria leptostachya Benth.

Shrub; Fl. and Fr.: October - November

Dahod Dt.: Kanvara dungar, Padaliya (Bedi, 1968); Dang Dt. (Tadvi, 2013): Ahwa, Pipaldahad (Suryanarayana, 1968); Devbhumi dwarka Dt.: Abhapar, Kileshwar, Venu, Ghumli (Nagar, 2005); Gir somnath Dt. (Sisodia, 2007); Junagadh Dt.: Junvaniya, Sasan (Santapau, 1962); Kheda Dt.: Shamariya; Narmada Dt.: Kalvat, Shamariya (Pradeepkumar, 1993); Navsari Dt.: Bansda; Porbandar Dt.: Godhana, Satvirda, Adityana, Ranavav (Nagar, 2005); Tapi Dt.: Vyara; Valsad Dt.: Hedri, Kaprada (Rao, 2012)

Crotalaria notonii Wight and Arn.

Shrub; Fl. and Fr.: September - November

Kachchh Dt. (Patel et al., 2011); Surat Dt.: Dumas

Crotalaria pusilla DC.

Herb; Fl. and Fr.: September - October

Chhota udepur Dt.: Kavant, Luni; Sabarkantha Dt.: Bavsar (Saxton and Sedgwick, 1918)

Crotalaria vestita Baker

Herb; Fl. and Fr.: October - December

Arvalli Dt.: Shamlaji; Dang Dt. (Tadvi, 2013): Malegaon, Saputara (Suryanarayana, 1968); Devbhumi dwarka Dt.: Abhapar, Kileshwar, Venu, Ghumli (Nagar, 2005); Jamnagar Dt.: Motwa Matli (Santapau, 1962); Junagadh Dt.: Bordevi (Santapau, 1962); Porbandar Dt.: Godhana, Satvirda, Adityana, Ranavav (Nagar, 2005); Valsad Dt.

*Dalbergia horrida (Dennst.) Mabb. [= Amerimnon horridum Dennst.]

Climbing shrub; Fl. and Fr.: January - April

Dahod Dt.: Devgadh baria, Ratanmahal (Bedi, 1968)

Derris heyneana (Wight and Arn.) Benth. [= Pongamia heyneana Wight and Arn.]

Climbing shrub; Fl. and Fr.: June - July

Gujarat (Singh et. al., 2015)

Desmodiastrum belgaumense (Wight) A.Pramanik and Thoth. [= Alysicarpus belgaumensis Wight]

Herb; Fl. and Fr.: September - October

Panchmahal Dt.: Pavagadh (Oza, 1961) 
*Desmodiastrum racemosum var. rotundifolium (Baker) A.Pramanik and Thoth. [= Desmodium rotundifolium Baker; Alysicarpus beddomei Schindl.; Desmodium ritchie Sanjappa]

Herb; Fl. and Fr.: August - September

Banaskantha Dt.: Balaram-Ambaji wildlife sanctuary (Meena, 2012); Dang Dt. (Sanjappa, 1977; Tadvi, 2013): Malegaon, Saputara (Suryanarayana, 1968), Subir; Gir somnath Dt. (Santapau, 1962; Sisodia, 2007); Panchmahal Dt.: Pavagadh (Oza, 1961)

Flemingia tuberosa Dalzell [= Moghania tuberosa (Dalzell) Kuntze]

Herb; Fl. and Fr.: September

Gujarat (Sanjappa, 1992); Valsad Dt.: Arnala (Patel, 2013)

*Geissaspis tenella Benth.

Herb; Fl. and Fr.: August - December

Dang Dt. (Tadvi, 2013)

Hardwickia binata Roxb.

Tree; Fl. and Fr.: October - February

Ahmedabad Dt. (Meena, 2014a); Anand Dt. (Anjaria, 2002); Chhota udepur Dt.: Kavant (Thaker, 1974), Lachhras (Desai, 2002), Hampeshwar (DNT1051, Thakar, 1970); Dang Dt.: Waghai; Gir somnath Dt. (Sisodia, 2007); Junagadh Dt.: Girnar (Santapau, 1962); Panchmahal Dt.: Shivrajpur; Surat Dt.: Cotton Research Farm nursery (Joshi, 1980); Valsad Dt.: Parnera (More, 1972)

Indigofera angulosa Edgew.

Shrub; Fl. and Fr.: August - October

Junagadh Dt.: Gir forest (Sanjappa, 1977); Narmada Dt.: Gora, Junaraj (Patel, 1971), Lal-Darvaja, Thevadia (Shah and Patel, 1971); Sabarkantha Dt.: Khedbrahma (Bhatt, 1971; Sanjappa, 1977), Derol Jagir (Parmar, 2012)

Indigofera coerulea var. monosperma (Santapau) Santapau [= Indigofera articulata var. monosperma Santapau]

Shrub; Fl. and Fr.: September - October

Gujarat (Sanjappa, 1992); Devbhumi dwarka Dt.: Dwarka railway line, Okha; Gir Somnath Dt.: Veraval (Gopal, 1983); Jamnagar Dt. (Malhotra and Wadhwa, 1973): Latipur (Rao, 2002), Laloi, Ranjit Sagar (Santapau, 1962), Vijarkhi (Nagar, 2005), Jamjodhpur; Junagadh Dt.: Petwad (Menon, 1979), on Rajkot road; Kachchh Dt. (Shah, 1978; Patel et al., 2011): Bhadreshwar (Rao, 1981), Mindhiyari (Pandey et al., 2009), Tapkeshwari (Joshi et al., 2012); Patan Dt.; Porbandar Dt.: Ranavav, Barda; Rajkot Dt.: Hingolgadh (Menon, 1979), Chibhda, Gondal, Jetalsar, Khirsara, Pradhyuman Park (Santapau, 1962; Nagar, 2008); Sabarkantha Dt.: Bhaderesar (Parmar, 2012); Surendranagar Dt.: Chotila (Santapau, 1962), Jupara

*Indigofera dalzellii T.Cooke [= Indigofera triquetra Dalzell]

Herb; Fl. and Fr.: July - September

Narmada Dt.: Shoolpaneshwar

Indigofera prostrata Willd.

Herb; Fl. and Fr.: August - December

Dahod Dt.: Ratanmahal (Bedi, 1968); Dang Dt. (Tadvi, 2013): Malegaon, Saputara, Subir (Suryanarayana, 1968); Valsad Dt. (Vora, 1980): Viraxet (Rao, 2012), Ghadoi (Patel RM, 1971)

*Indigofera uniflora Roxb.

Herb; Fl. and Fr.: July - March

Gujarat (Singh et. al., 2015)

Mimosa prainiana Gamble

Tree; Fl. and Fr.: August - December

Gujarat (Singh et. al., 2015)

Pterocarpus marsupium subsp. acuminatus (Prain) Thoth. [= Pterocarpus marsupium var. acuminatus Prain]

Tree; Fl. and Fr.: April - July

Banaskantha Dt.: Jessore (Punjani et al., 2015); Bhavnagar Dt.: Kadambagiri (Meena, 2014b); Chhota udepur Dt.: Alwa (Thaker, 1974), Mithibor at Dolariya (Desai, 2002); Dahod Dt.: Ratanmahal (Bedi, 1968); Dang Dt. (Tadvi, 2013): Ahwa, Subir (Suryanarayana, 1968); Devbhumi dwarka Dt.: Abhapar, Ghumli, (Nagar, 2005); Jamnagar Dt.: Laloi (Santapau, 1962); Junagadh Dt. (Santapau, 1962); Narmada Dt.: Junaraj, Mathavali, Ninaighat (Pradeepkumar, 1993), Kevadiya; Panchmahal Dt. (Bhatt, 1975); Porbandar Dt.: Barda (Thaker, 1910), Godhana, Satvirda (Nagar, 2005); Sabarkantha Dt.: Khedbrahma (Bhatt, 1971), Damavas (Bhatt and Sabnis, 1972); Valsad Dt. (Inamdar and Patel, 1971; Vora, 1980): Parnera (More, 1972), Marala (Patel RM, 1971), Hedri

Senna montana (Roth) V.Singh [= Cassia montana Roth]

Shrub; Fl. and Fr.: July - October 
Devbhumi dwarka Dt.: Kileshwar; Junagadh Dt. (Santapau, 1962): Bordevi, Ramnath; Porbandar Dt.: Barda (Thaker, 1910), Naliadhar, Satvirda

Sesbania procumbens (Roxb.) Wight and Arn. [= Aeschynomene procumbens Roxb.]

Herb; Fl. and Fr.: February - March

Vadodara Dt. (Sabnis, 1967)

Smithia setulosa Dalzell

Herb; Fl. and Fr.: September - October

Dang Dt. (Tadvi, 2013): Saputara (Suryanarayana, 1968); Valsad Dt.: Dharampur

Tephrosia collina V.S.Sharma

Shrub; Fl. and Fr.: August - October

Gujarat (Sanjappa, 1992); Jamnagar Dt.: Jamjodhpur (Nagar, 2007; Bhambra, 2015); Kachchh Dt.: Adesar (Raghavan et al., 1981); Mehsana Dt.: Salipur, Vadnagar; Narmada Dt.: Rajpipla (Shah, 1978), Junaraj; Sabarkantha Dt.: Dhandha (Parmar, 2012)

Tephrosia jamnagarensis Santapau [= Tephrosia axillaris A.R.Sm.]

Herb; Fl. and Fr.: September - October

Gujarat (Sanjappa, 1992); Bharuch Dt.: Bhadbhut (Vyas, 1973; Shah, 1978); Jamnagar Dt.: Victoria bridge, near Rozi island (Santapau, 1962), Khadkhambhaliya vidi (Nagar et. al., 2003; Bhambra, 2015), Rasi forest, RojiValsura; Junagadh Dt.: Sagdividi; Surat Dt.: Udhna (Joshi, 1980)

Vigna khandalensis (Santapau) Sundararagh. and Wadhw [= Phaseolus khandalensis Santapau]

Herb; Fl. and Fr.: September - October

Dang Dt. (Tadvi, 2013): Saputara (Suryanarayana, 1968); Valsad Dt.: Kaprada

\section{Euphorbiaceae Juss:-}

*Acalypha malabarica Müll.Arg. [= Ricinocarpus malabaricus (Müll.Arg.) Kuntze]

Herb; Fl. and Fr.: July - October

Ahmedabad Dt. (Meena, 2014a); Banaskantha Dt.: Palanpur (Meena, 2012); Bhavnagar Dt. (Oza, 1991); Chhota udepur Dt.: Kavant (Thaker, 1974); Gir somnath Dt. (Santapau and Raizada, 1954); Junagadh Dt.: Sasan (Bole and Pathak, 1988; Baxi, 2003); Rajkot Dt.: Gondal irrigation lake, Pradhyuman park (Bole and Pathak, 1988); Sabarkantha Dt. (Parmar, 2012): Damavas (Bhatt, 1971)

Euphorbia coccinea B.Heyne ex Roth [= Chamaesyce coccinea (B.Heyne ex Roth) Soják]

Herb; Fl. and Fr.: June - October

Daman: Daman airport, Delvada (29.6.1964, M.Y. Ansari 98538, BSI)

*Euphorbia deccanensis V.S.Raju

Herb; Fl. and Fr.: October

Devbhumi dwarka Dt.: Beyt; Jamnagar Dt.

Euphorbia elegans Spreng. [= Chamaesyce elegans (Spreng.) Soják]

Herb; Fl. and Fr.: Mar - May

Porbandar Dt.: Bhad, Garej, Kadachh, Madhavpur (Bole and Pathak, 1988; Baxi, 2003)

*Euphorbia perbracteata Gage [= Tithymalus perbracteatus (Gage) Soják]

Herb; Fl. and Fr.: December - April

Anand Dt.: Dhuvaran; Bharuch Dt.: Kavi; Daman: Dabel near tank (4.6.1963, R.S. Rao 89002, CAL); Dang Dt. (Tadvi, 2013): Malegaon, Saputara; Surat Dt. (Joshi, 1980); Vadodara Dt.: Savli (Padate, 1973), Chokari

Euphorbia pycnostegia Boiss. [= Euphorbia pycnostegia var. pycnostegia]

Herb; Fl. and Fr.: October - December

Dang Dt. (Tadvi, 2013): Saputara (Suryanarayana, 1968), Malegaon; Junagadh Dt.: Visavadar (Bole and Pathak, 1988; Baxi, 2003); Rajkot Dt. (Bole and Pathak, 1988)

*Euphorbia pycnostegia var. zornioides (Boiss.) Santapau [= Euphorbia zornioides Boiss.]

Herb; Fl. and Fr.: August - October

Daman: Savardem, Valpai road (3.10.1970, N.P. Singh, 124958 BSI); Surat Dt.: Bhimpor, Dumas, Magdalla; Vadodara Dt.: Pratapnagar (Patil and Sabnis, 1982)

Euphorbia sebastinei Binojk. and N.P.Balakr. [= Chamaesyce sebastinei (Binojk. and N.P.Balakr.) V.S.Raju]

Herb; $F l$. and Fr.: March - June

Devbhumi dwarka Dt. (Binojkumar, 1993)

*Homonoia retusa (Graham ex Wight) Müll.Arg. [= Adelia retusa Graham ex Wight]

Shrub; Fl. and Fr.: November - April

Narmada Dt.: Gora, Kevadia (Patel, 1971), Surpan 
*Mallotus polycarpus (Benth.) Kulju and Welzen [= Trewia polycarpa Benth.; Trewia nudiflora var. polycarpa (Benth.) Susila and N.P.Balakr.]

Tree; Fl. and Fr.: December - June

Narmada Dt.; Dang Dt.: Ahwa; Valsad Dt.: Barpuda, Gundiya, Pangarbari, Kaprada (Reddy, 1987), Amba (Rao, 2012), Jogvel, Lavkar, Ozarda, Sildha (Vora, 1980)

Malpighiaceae Juss;-

*Aspidopterys cordata (B.Heyne ex Wall.) A.Juss. [= Hiraea cordata B.Heyne ex Wall.]

Climber; Fl. and Fr.: September - January

Dang Dt. (Chavan and Oza, 1966): Saputara (Patel, 2013), Ahwa, Malegaon, Pipaldahad, Shamgahan (Suryanarayana, 1968); Navsari Dt.: Mankunia; Valsad Dt.: Pangarbari, Sutharpada (Reddy, 1987; Patel, 2013)

Phyllanthaceae Martinov:-

*Bridelia montana (Roxb.) Willd. [= Clutia montana Roxb.]

Shrub; Fl. and Fr.: August - January

Valsad Dt.: Amba, Avdha, Lavkar, Mandva, Nandgam, Sanjan, Sildha, Umbergaon

*Phyllanthus lawii J.Graham [= Diasperus lawii (J.Graham) Kuntze; Phyllanthus juniperinoides Müll.Arg.]

Shrub; Fl. and Fr.: November

Bharuch Dt.: Kabirvad; Chhota udepur Dt.: Hampheshwar; Narmada Dt.: Garudeshwar, Kevadiya

Salicaceae Mirb:-

*Flacourtia montana J.Graham

Tree; Fl. and Fr.: December - January

Dang Dt.: Ahwa, Mahal, Malegaon; Gir somnath Dt. (Sisodia, 2007); Junagadh Dt.; Panchmahal Dt.: Pavagadh (Oza, 1961; Chavan and Oza, 1962), Narukot

Rhamnaceae Juss:-

*Ziziphus caracutta Buch.-Ham. ex Roxb.

Tree; Fl. and Fr.: April - June

Dang Dt.: Malegaon; Junagadh Dt.: Sasan (Hiran river); Valsad Dt.

*Ziziphus horrida Roth

Shrub; Fl. and Fr.: July - December

Ahmedabad Dt. (Meena, 2014a): Kadi (Yogi, 1970); Amreli Dt.: Dhari (Menon, 1979); Banaskantha Dt.: Ambaji (Meena, 2012); Dang Dt.: Ahwa (Suryanarayana, 1968); Gir somnath Dt.: Una (Menon, 1979); Junagadh Dt.: Ramnath, Visavadar (Menon, 1979); Mahisagar Dt.: Lunawada; Mehsana Dt. (Shah and Yogi, 1974); Narmada Dt.: Kevadiya (Patel, 1971); Panchmahal Dt. (Bhatt, 1975): Pavagadh (Oza, 1961); Surat Dt.: Chandrapada, Umarpada

Ziziphus williamii Bhandari and Bhansali

Tree; Fl. and Fr.: August - October

Ahmedabad Dt.; Bharuch Dt.; Devbhumi dwarka Dt.: Beyt, Shankhodhar island; Kachchh Dt.: Rudramata

\section{Brassicaceae Burnett:-}

*Rorippa cochlearioides (Roth) Al-Shehbaz and Jonsell [= Alyssum cochlearioides Roth; Cochlearia cochlearioides (Roth) Santapau and Mahesw.]

Herb; Fl. and Fr.: December - January

Chhota udepur Dt.: Hampheshwar (Thaker, 1974), Moti Chikli, Ambadungar; Narmada Dt.: Chopadi, Kalvat, Ninaighat (Pradeepkumar, 1993), Gora, Kevadiya (Patel, 1971), Suryakhadi (Shah and Patel, 1971), Rajpipla; Panchmahal Dt.: Pavagadh

Cleomaceae Bercht. and J. Presl:-

Cleome simplicifolia Hook.f. and Thomson

Herb; Fl. and Fr.: August - September

Bhavnagar Dt. (Oza, 1991): Palitana (Menon, 1979), Hathab, Shatrunjaya dam (Meena, 2014b); Chhota udepur Dt. (Desai, 2002): Kavant (Thaker, 1974); Dahod Dt.: Ratanmahal (Bedi, 1968), Pipargota (BEDI2162 Bedi, 1962), Tokarva (BEDI3396 Bedi, 1962), Devgadh Baria; Devbhumi dwarka Dt.: Bhanvad (Santapau, 1962); Gandhinagar Dt.: Mansa; Gir somnath Dt. (Sisodia, 2007); Jamnagar Dt.: Vijarkhi dam (Santapau, 1962), Abhapar, Kileshwar, Venu, Ghumli (Nagar, 2005); Junagadh Dt.: Sapnes, Sasan (Menon, 1979); Kachchh Dt. (Patel et al., 2011): 
Nakhatrana (Bhatt, 1993), Mindhiyari (Pandey et al., 2009); Kheda Dt.: Matar (BEDI772 Bedi, 1960); Panchmahal Dt.: Chari (BEDI1906 Bedi, 1961); Porbandar Dt.: Godhana, Satvirda, Adityana, Ranavav (Thaker, 1910; Nagar, 2005); Rajkot Dt.: Hingolgadh (Menon, 1979), Gondal, Pradhyuman park (Santapau, 1962); Sabarkantha Dt.: Dan Mahudi (RPB1463, Bhatt, 1968), Khedbrahma (Yogi, 1970; Bhatt, 1971; Parmar, 2012); Surat Dt.: Rander (Joshi, 1980); Vadodara Dt.: Savli (Padate, 1969; Padate, 1973), Wasanpura (SNP1605, Padate, 1961), Lacchanpur (SNP701, Padate, 1959), Mokshi (SNP3867, Padate, 1972), Manjusar (SNP1301, Padate, 1960)

\section{Malvaceae Juss:-}

Abelmoschus manihot subsp. tetraphyllus var. megaspermus Hemadri [= Abelmoschus manihot var. megaspermus Hemadri]

Undershrub; Fl. and Fr.: October - November

Chhota udepur Dt. (Desai, 2002): Hampheshwar; Mehsana Dt.: Kadi

Abelmoschus tuberculatus var. deltoidefolius T.K.Paul and M.P.Nayar

Undershrub; Fl. and Fr.: August - October

Kachchh Dt.: Narayan sarovar (Parmar and Singh, 2003), Mindhiyari (Pandey et al., 2009)

*Erinocarpus nimmonii J.Graham [= Erinocarpus nimmoanus Mast.]

Tree; Fl. and Fr.: September - February

Dang Dt: Waghai; Valsad Dt.

Eriolaena stocksii Hook.f. and Thomson ex Mast.

Tree; Fl. and Fr.: June - August

Dang Dt. (Tadvi, 2013): Waghai (Gopal, 1983), Gira, Giri, Taola hills (Suryanarayana, 1968), Saputara (Shah and Suryanarayana, 1968), Kalibel; Junagadh Dt.: Sapnes (Menon, 1979); Narmada Dt.: Rajpipla (Gopal, 1983), Gora, Piplod, Sagai (Sharma, 2010), Thavadia; Valsad Dt.

\section{Combretaceae R. Br:-}

Anogeissus sericea var. nummularia King ex Duthie [= Anogeissus rotundifolia Blatt. and Hallb.]

Tree; Fl. and Fr.: January - April

Arvalli Dt.: Mahudi, Modasa, Vatrak; Banaskantha Dt.: Balaram temple (Rajendrakumar and Kalavathy, 2010; Meena, 2012), Jessore wildlife sanctuary (Rajendrakumar and Kalavathy, 2010); Gandhinagar Dt.: Mansa; Kheda Dt.; Mehsana Dt.: Juna Sudarsana, Taranga hill (Rajendrakumar and Kalavathy, 2010); Panchmahal Dt.: Tuwa, Pavagadh; Sabarkantha Dt.: Modasa (Parmar, 2012); Vadodara Dt.: Karachiya (Rao, 2002)

Anogeissus sericea Brandis

Tree; Fl. and Fr.: January - April

Ahmedabad Dt. (Meena, 2014a); Anand Dt. (Anjaria, 2002); Arvalli Dt.: Mahudi (Yogi, 1970), Modasa, Vatrak (Saxton and Sedgwick, 1918); Banaskantha Dt.: Jessore (Meena, 2012; Punjani et al., 2015); Gandhinagar Dt.: Mansa (Yogi, 1970), Moti Pavathi (Gopal, 1983); Junagadh Dt.: Ghodawadi (Menon, 1979); Panchmahal Dt.: Pavagadh (Shah, 1978), Tuwa (Deshpande, 1968); Sabarkantha Dt.: Khedbrahma (Bhatt, 1971), Mama na pipla (Bhatt and Sabnis, 1972)

\section{Lythraceae J. St.-Hil:-}

Ammannia nagpurensis T.Mathew and M.P.Nayar

Herb; $F l$. and Fr.: October - November

Junagadh Dt.: Sasangir to Junvaniya (Mathew and Nayar, 1989); Navsari Dt.: Unai (Mathew and Nayar, 1989)

*Lagerstroemia microcarpa Wight

Tree; Fl. and Fr.: May - December

Ahmedabad Dt. (Meena, 2014a); Chhota udepur Dt.: Kadipani, Adtia, Satun, Raipur Kundai, Ghantoli-songir (Desai, 2002), Kavant (Thaker, 1974); Dang Dt. (Suryanarayana, 1968); Narmada Dt.: Junaraj, Kevadiya (Patel, 1971), Kelda, Kokati, Ninaighat, Sagai, Shisha; Valsad Dt.: Bildha, Jogvel, Nana Pondha, Panas (Vora, 1980), Hedri, Pendha, Umli (Rao, 2012)

\section{Myrtaceae Juss:-}

*Syzygium salicifolium (Wight) J.Graham [= Eugenia salicifolia Wight; Syzygium heyneanum (Duthie) Gamble; Eugenia heyneana Duthie]

Tree; Fl. and Fr.: April - May

Arvalli Dt.: Modasa, Vatrak (Saxton and Sedgwick, 1918), Mahudi; Banaskantha Dt.: Gannapipadi, Ambaji (Meena, 2012); Chhota udepur Dt.: Kavant (Thaker, 1974); Dahod Dt.: Ratanmahal (Bedi, 1968), Bhuvera (Gopal, 1983); 
Dang Dt. (Tadvi, 2013); Gandhinagar Dt. (Meena, 2014a); Gir somnath Dt. (Sisodia, 2007); Junagadh Dt. (Menon, 1979); Narmada Dt.: Rajpipla (Gopal, 1983), Kelda, Sagai, Waghumar (Pradeepkumar, 1993); Panchmahal Dt.; Sabarkantha Dt.: Khedbrahma (Yogi, 1970; Bhatt, 1971; Bhatt and Sabnis, 1972), Pahada (Yogi, 1970), Vijainagar, Dholwani (Parmar, 2012); Surat Dt.: Zankhvav (Yadav, 1979); Vadodara Dt.: Kamalpura; Valsad Dt. (Vora, 1980): Hedri, Pendha (Yadav, 1979), Kaprada (Rao, 2012)

\section{Burseraceae Kunth:- \\ *Boswellia serrata Roxb. ex Colebr.}

Tree; Fl. and Fr.: December - April

Ahmedabad Dt.: Kundal; Arvalli Dt.: Modasa; Banaskantha Dt.: Jessore (Meena, 2012), Dantiwada, Palanpur (Patel, 2009); Bharuch Dt.; Bhavnagar Dt.: Palitana, Shatrunjaya hill (Meena, 2014b); Chhota udepur Dt.: Kevdi, Kundal, Satun (Desai, 2002), Kavant (Thaker, 1974), Ambadungar (DNT1518, Thakkar, 1971); Dahod Dt.: Ratanmahal (Bedi, 1968), Devgadh Baria (Chavan et al., 1963), Dhanpur (BEDI1222, Bedi, 1961); Dang Dt. (Tadvi, 2013): Subir (Suryanarayana, 1968); Gir somnath Dt. (Santapau and Raizada, 1954; Sisodia, 2007); Jamnagar Dt.: Laloi (Santapau, 1962); Junagadh Dt.: Sapnes, Tulsishyam (Menon, 1979), Kankai (Santapau, 1962), Sasan (Gopal, 1983); Kachchh Dt. (Patel et al., 2011); Kheda Dt.: Matar (BEDI2423 Bedi, 1962); Mahisagar Dt.; Narmada Dt.: Gora, Junaraj (Patel, 1971), Dhirkhadi, Mokhdi, Surpan, Thavadia (Pradeepkumar, 1993); Navsari Dt.; Panchmahal Dt.: Machi, Pavagadh (Oza, 1961); Patan Dt.: Satun; Rajkot Dt.: Hadala (Menon, 1979); Sabarkantha Dt.: Khedbrahma (Bhatt, 1971), Idar (Yogi, 1970), Kotda (Parmar, 2012), Modasa (Saxton and Sedgwick, 1918; Parmar, 2012); Surat Dt.: Kevdi; Surendranagar Dt.: Chotila (Santapau, 1962), Rampara; Valsad Dt.: Chavshala (Yadav, 1979), Dharampur (Gopal, 1983; Reddy, 1987), Kaprada, Nana Pondha (Vora, 1980)

\section{Amaranthaceae Juss:-}

*Achyranthes coynei Santapau

Shrub; Fl. and Fr.: September - March

Kachchh Dt.: Kalo Dungar (Satish et al., 2015)

\section{Tamaricaceae Link:-}

Tamarix kutchensis B.V.Shetty and R.P.Pandey

Shrub; Fl. and Fr.: November - January

Kachchh Dt.: Mundra - Mandvi (Shetty and Pandey, 1992), Narayan sarovar tank side (Pandey et al., 2009)

\section{Loranthaceae Juss:-}

Dendrophthoe falcata var. coccinea (Talbot) Santapau [= Loranthus longiflorus var. coccinea Talbot]

Shrub; Fl. and Fr.: February - March

Arvalli Dt.: Modasa; Chhota udepur Dt.: Kavant (Thaker, 1974); Junagadh Dt.: Girnar (Bole and Pathak, 1988); Narmada Dt.: Rajpipla (Shah, 1967); Valsad Dt.: Kaprada (Rao, 2012)

\section{Balsaminaceae A. Rich:-}

*Impatiens minor (DC.) Bennet [= Balsamina minor DC.; Impatiens kleinii Wight and Arn.]

Herb; Fl. and Fr.: August - November

Dahod Dt.: Banvaro (BEDI457, Bedi, 1961; BEDI3012 Bedi, 1962), Pipargota (BEDI1439, Bedi, 1961); Dang Dt. (Tadvi, 2013): Ahwa, Malegaon (Suryanarayana, 1968); Mehsana Dt.: Medha; Narmada Dt.: Shisha (Pradeepkumar, 1993); Navsari Dt.: Bansda, Vati; Tapi Dt.: Gaumukh, Vyara; Valsad Dt.: Pangarbari (Reddy, 1987), Ozarda (Rao, 2012), Bilpudi, Boralai

Boraginaceae Juss:-

*Adelocaryum coelestinum (Lindl.) Brand [= Cynoglossum coelestinum Lindl.; Paracaryum coelestinum (Lindl.) C.B.Clarke]

Herb; Fl. and Fr.: September - November

Dang Dt. (Tadvi, 2013): Don, Saputara; Narmada Dt.: Shisha (Pradeepkumar, 1993), Ninaighat

* Cordia domestica Roth

Tree; Fl. and Fr.: May - August

Ahmedabad Dt. (Meena, 2014a); Chhota udepur Dt. (Shah, 1978): Koraj (Desai, 2002); Dahod Dt.: Popatkuwa (Rao, 2002); Dang Dt. (Tadvi, 2013): Mahal (Yadav, 1979); Junagadh Dt.: Mangrol (Santapau, 1953; Bole and 
Pathak, 1988); Panchmahal Dt. (Shah, 1978); Sabarkantha Dt.: Bayad (Parmar, 2012); Valsad Dt.: Kaprada (Reddy, 1987)

Apocynaceae Juss:-

Brachystelma laevigatum Hook.f. [= Eriopetalum laevigatum Wight]

Herb; Fl. and Fr.: August

Kachchh Dt.

Ceropegia odorata Nimmo [= Ceropegia blatteri McCann]

Climber; $F l$. and Fr.: July - August

Panchmahal Dt.: Pavagadh (Sabnis and Bedi, 1971); Sabarkantha Dt.: Vijaynagar

Sarcostemma intermedium Decne.

Shrub; Fl. and Fr.: July - September

Bhavnagar Dt.: Piram island at the mouth of the Narmada river

*Hemidesmus indicus var. pubescens (Wight and Arn.) Hook.f. [= Hemidesmus pubescens Wight and Arn.]

Climber; Fl. and Fr.: September - October

Junagadh Dt.: Girnar, Chobari (Patel, 2013); Kachchh Dt.: Muru village along Hajipir road (Singhadiya et al., 2011); Narmada Dt.: Ninai waterfall; Panchmahal Dt.

*Heterostemma dalzellii Hook.f.

Shrub; Fl. and Fr.: August - October

Narmada Dt.: Gichad (Meena and HariKrishna, 2015)

Tylophora dalzellii Hook.f.

Climber; Fl. and Fr.: April - December

Saurashtra (Bole and Pathak, 1988); Dahod Dt.: Dhanpur; Dang Dt. (Tadvi, 2013): Malegaon (Suryanarayana, 1968), Ahwa (MCJ500, Joshi, 1970); Devbhumi dwarka Dt.: Abhapar, Kileshwar, Venu, Ghumli (Nagar, 2005); Junagadh Dt.: Sasan; Kachchh Dt. (Thaker, 1910); Patan Dt.: Satun; Porbandar Dt.: Barda (Thaker, 1910; Nagar, 2005); Valsad Dt.: Pangarbari, Sutharpada (Patel, 2013), Dharampur

Wrightia dolichocarpa Bahadur and Bennet

Tree; Fl. and Fr.: December - April

Banaskantha Dt: Jessore (Rao, 2002); Dang Dt. (Tadvi, 2013); Dadra and Nagar Haveli (Reddy, 1987): Bonta, Saili (Bahadur and Bennet, 1978); Devbhumi dwarka Dt.: Ghumli; Junagadh Dt.: Girnar; Narmada Dt.: Sagai; Sabarkantha Dt.: Vijaynagar; Valsad Dt.: Moti Korval, Kapurnya (Reddy, 1987), Wilson hill

\section{Gentianaceae Juss:-}

* Canscora concanensis C.B.Clarke

Herb; Fl. and Fr.: October - November

Dang Dt. (Tadvi, 2013): Ahwa (Suryanarayana, 1968); Devbhumi dwarka Dt.: Abhapar, Venu (Nagar, 2005); Mahisagar Dt.: Lunawada; Navsari Dt.: Ambabari (Desai, 1976); Panchmahal Dt. (Bhatt, 1975): Machi, Bhadrakali (Bedi et al., 1968); Porbandar Dt.: Godhana, Satvirda (Nagar, 2005); Surat Dt.: Siddh Kutir (Joshi, 1980)

*Exacum pumilum Griseb.

Herb; Fl. and Fr.: August - November

Dang Dt. (Tadvi, 2013): Ahwa (Suryanarayana, 1968); Jamnagar Dt.: Khatiya, Ranjit Sagar; Junagadh Dt.: Gir (Sisodia, 2007); Navsari Dt.: Bansda (Desai, 1976); Valsad Dt. (Patel RM, 1971): Kaprada, Nana Pondha (Vora, 1980), Pardi (More, 1972)

*Swertia minor (Griseb.) T.Cooke

Herb; Fl. and Fr.: August

Dang Dt. (Tadvi, 2013): Malegaon (Suryanarayana, 1968); Valsad Dt.: Pangarbari, Pindval

\section{Rubiaceae Juss:-}

*Ixora brachiata Roxb.

Tree; Fl. and Fr.: February - June

Banaskantha Dt.: Balaram-Ambaji wildlife sanctuary (Meena, 2012); Bhavnagar Dt. (Oza, 1991); Chhota udepur Dt.: Ganiyar Bari, Jetpur Pavi, Koraj, Naswadi, Satun, Vasangadh (Desai, 2002); Dang Dt. (Tadvi, 2013); Gir somnath Dt. (Sisodia, 2007); Junagadh Dt.: Girnar, Mithyala, Sapnes (Menon, 1979), Sasan, Willingdon dam (Santapau, 1962); Mahisagar Dt.: Dezar, Shingnali (Bhatt, 1975); Mehsana Dt. (Shah and Yogi, 1974); Porbandar Dt.: Kileshwar (Santapau, 1962); Rajkot Dt.: Hadala (Menon, 1979), Gondal lake (Santapau, 1962); Valsad Dt.: Kaprada (Gopal, 1983), Dhamni, Pendha (Yadav, 1979), Bilpudi, Mandva, Nana Pondha (Vora, 1980) 
Kohautia nagporensis (Brace ex Haines) Santapau and Merchant [= Oldenlandia nagporensis Brace ex Haines] Herb; Fl. and Fr.: August - September

Panchmahal Dt.

*Neanotis rheedei (Wight and Arn.) W.H.Lewis [= Hedyotis rheedei Wight and Arn.]

Herb; Fl. and Fr.: July - October

Chhota udepur Dt.; Dang Dt. (Chavan and Oza, 1966; Tadvi, 2013); Valsad Dt.: Dharampur, Kaprada, Nana Pondha (Vora, 1980)

*Pavetta crassicaulis Bremek.

Shrub; Fl. and Fr.: July - October

Dang Dt. (Tadvi, 2013): Waghai (Yadav, 1979), Malegaon, Saputara (Suryanarayana, 1968); Valsad Dt.: Kaprada (Yadav, 1979), River Par, River Kolak (Vora, 1980), Dharampur, Dixal, Huda

\section{Acanthaceae Juss:- \\ *Asystasia dalzelliana Santapau}

Herb; Fl. and Fr.: August - December

Dang Dt. (Tadvi, 2013): Ahwa, Malegaon, Saputara, Subir (Suryanarayana, 1968)

*Barleria cuspidata F.Heyne ex Nees

Shrub; Fl. and Fr.: December - March

Dang Dt.; Gandhinagar Dt.: Norda nala; Gir somnath Dt. (Sisodia, 2007); Kachchh Dt.; Mehsana Dt. (Shah and Yogi, 1974)

*Barleria gibsonii Dalzell

Undershrub; Fl. and Fr.: September - November

Dang Dt.: Saputara (Suryanarayana, 1968), Gira; Gir somnath Dt. (Sisodia, 2007); Junagadh Dt.: Girnar (Menon, 1979); Valsad Dt.: Dharampur, Kaprada, Nana Pondha (Vora, 1980), Rabda (More, 1972), Fansa, Pangarbari, Sarigam

*Barleria lawii T.Anderson [= Barleria sepalosa C.B.Clarke]

Shrub; Fl. and Fr.: October - December

Bhavnagar Dt. (Menon, 1979); Dang Dt.: Saputara (Suryanarayana, 1968); Junagadh Dt.: Cremation ground (Bole and Pathak, 1988), Girnar; Valsad Dt.: Dharampur, Kaprada, Nana Pondha (Vora, 1980)

*Barleria prattensis Santapau Undershrub; $F l$. and Fr.: September - December

Banaskantha Dt.: Koteshwar near Ambaji (Meena, 2012); Bhavnagar Dt.: Kadambagiri (Meena, 2014b); Chhota udepur Dt.: Vagasthal, Rajmahal, Kadipani, Ambadungar, Satun, Jamlidam, Naswadi, Turkheda (Desai, 2002), Kavant (Thaker, 1974); Dahod Dt.: Alindra, Pipargota (Bedi, 1968); Dang Dt. (Tadvi, 2013): Borkhal, Mahal, Waghai (Yadav, 1979), Ahwa, Malegaon, Subir (Suryanarayana, 1968), Don; Gir somnath Dt. (Santapau and Raizada, 1954); Jamnagar Dt.: Kileshwar (Bole and Pathak, 1988); Junagadh Dt.: Sasan (Menon, 1979), Girnar, Junvaniya (Bole and Pathak, 1988); Mehsana Dt. (Shah and Yogi, 1974); Narmada Dt.: Mohbi, Namgir, Waghumar (Pradeepkumar, 1993); Panchmahal Dt.: Pavagadh (Oza, 1961), Tuwa (Deshpande, 1968); Sabarkantha Dt. (Parmar, 2012): Khedbrahma (Bhatt, 1971); Vadodara Dt.: Savli (Padate, 1969; Padate, 1973); Valsad Dt.: Avdha, Dhamni, Pendha, Sidhumbar, Sanjan, Palgam (Yadav, 1979), Amba, Bilpudi, Mandva (Vora, 1980), Parnera (More, 1972), Dungri hill (Patel RM, 1971), Khadakval (Rao, 2012)

*Cynarospermum asperrimum (Nees) Vollesen [= Blepharis asperrima Nees]

Herb; Fl. and Fr.: September - December

Dang Dt.: Malegaon; Sabarkantha Dt. (Parmar, 2012): Khedbrahma (Bhatt, 1971); Valsad Dt.: Pendha (Yadav, 1979), Pangarbari

*Dyschoriste vagans (Wight) Kuntze [= Calophanes dalzellii Benth. ex T.Anderson; Calophanes vagans Wight] Herb; Fl. and Fr.: November - February

Dang Dt.: Bheskatri, Mahal, Malegaon, Saputara; Narmada Dt.; Valsad Dt. (Patel RM, 1971): Veri Bhavada (Rao, 2012), Dharampur, Kaprada, Nana Pondha (Vora, 1980), Nanakwada (Patel RM, 1971), Moti Korval, Tutarkhed

*Eranthemum roseum (Vahl) R.Br. [= Justicia rosea Vahl; Daedalacanthus roseus (Vahl) T.Anderson] Undershrub; Fl. and Fr.: October - January

Ahmedabad Dt.; Anand Dt. (Anjaria, 2002); Chhota udepur Dt.: Mogra, Kadipani, Hampeshwar road, Koraj, Satun, Raipur-kundal, Naswadi, Lagami (Desai, 2002), Kavant (Thaker, 1974); Dahod Dt.: Ratanmahal (Bedi, 1968); Dang Dt. (Tadvi, 2013): Ahwa, Mahal, Malegaon, Pipaldahad, Saputara, Subir (Suryanarayana, 1968), Bheskatri; Devbhumi dwarka Dt.: Abhapara, Ghumli (Bole and Pathak, 1988); Jamnagar Dt.; Junagadh Dt.: Girnar (Bole and Pathak, 1988), Sasan (Menon, 1979); Mehsana Dt. (Shah and Yogi, 1974); Narmada Dt.: Ninaighat, Sagai, Vav, 
Waghumar (Pradeepkumar, 1993), Rajpipla (Shah, 1967), Chanderpada; Navsari Dt.: Abrama; Panchmahal Dt.: Pavagadh (Oza, 1961); Sabarkantha Dt.: Khedbrahma (Bhatt, 1971; Bhatt and Sabnis, 1972); Vadodara Dt.: Savli (Padate, 1969), Kamalpura; Valsad Dt. (Inamdar and Patel, 1971): Vavar (Rao, 2012), Dharampur, Kaprada, Nana Pondha (Vora, 1980), Abrama, Atgam, Palan (Patel RM, 1971), Hedri, Parnera, Pendha

*Gantelbua urens (B.Heyne ex Roxb.) Bremek.

Herb; Fl. and Fr.: January - March

Chhota udepur Dt.: Kavant (Thaker, 1974); Dang Dt.: Ahwa; Narmada Dt.: Gichad, Khaman, Namgir (Pradeepkumar, 1993); Navsari Dt.: Bilimora, Unai; Sabarkantha Dt. (Parmar, 2012): Khedbrahma (Bhatt, 1971); Surat Dt. (Joshi, 1980); Vadodara Dt.: Sundarpura (Sabnis, 1967), Dabhoi; Valsad Dt. (Patel RM, 1971): Kaprada (Rao, 2012), Pardi (More, 1972), Dharampur, Nana Pondha

*Haplanthodes nilgherrensis (Wight) R.B.Majumdar [= Haplanthus nilgherrensis Wight.]

Herb; Fl. and Fr.: September - December

Banaskantha Dt.: Balaram-Ambaji wildlife sanctuary (Meena, 2012); Dahod Dt.: Banvaro, Panam (Bedi, 1968); Narmada Dt.: Namgir, Shisha, Waghumar (Pradeepkumar, 1993), Rajpipla (Shah, 1967); Panchmahal Dt.: Pavagadh (Oza, 1961); Surat Dt. (Joshi, 1980); Vadodara Dt.; Valsad Dt. (Inamdar and Patel, 1971): Dharampur, Kaprada, Nanapondha (Vora, 1980)

*Haplanthodes tentaculatus (L.) R.B.Majumdar [= Ruellia tentaculata L.]

Herb; Fl. and Fr.: October - January

Ahmedabad Dt.; Chhota udepur Dt.: Kavant (Thaker, 1974); Dang Dt. (Tadvi, 2013): Bheskatri, Pipaldahad (Yadav, 1979), Ahwa, Mahal, Malegaon, Subir (Suryanarayana, 1968); Gir somnath Dt. (Santapau and Raizada, 1954); Junagadh Dt.: Shirvaniya (Bole and Pathak, 1988); Mahisagar Dt.; Narmada Dt.: Sagai; Navsari Dt.: Bansda (Desai, 1976); Panchmahal Dt.: Vishwamitri riverbank (Chavan and Sabnis, 1960); Surat Dt.: Zankhvav (Yadav, 1979); Valsad Dt. (Patel RM, 1971): Kaprada (Rao, 2012), Dharampur, Nanapondha (Vora, 1980), Parnera (More, 1972), Hedri, Pendha (Yadav, 1979)

*Haplanthodes verticillatus (Roxb.) R.B.Majumdar

Herb; Fl. and Fr.: October - January

Banaskantha Dt.: Balaram-Ambaji road, Jessore wildlife sanctuary (Meena, 2012); Chhota udepur Dt.: Kavant (Thaker, 1974); Dahod Dt.: Ratanmahal (Bedi, 1968); Dang Dt. (Tadvi, 2013): Ahwa, Nilsakiya (Yadav, 1979), Malegaon, Pipaldahad, Saputara, Subir (Suryanarayana, 1968); Gir somnath Dt. (Santapau and Raizada, 1954); Junagadh Dt.: Girnar, Junvaniya, Kankai, Sasan, Shirvaniya (Bole and Pathak, 1988); Kheda Dt.: Kapadvanj; Narmada Dt.: Chopadi, Namgir, Vav (Pradeepkumar, 1993), Rajpipla (Shah, 1967); Navsari Dt.: Anklachh, Vati (Desai, 1976); Panchmahal Dt.: Machi (Oza, 1961), Kavant; Sabarkantha Dt.: Khedbrahma (Bhatt, 1971; Bhatt and Sabnis, 1972), Dholwani, Vanaj (Parmar, 2012); Surat Dt.: Vankal; Vadodara Dt.: Savli (Padate, 1969; Padate, 1973); Valsad Dt.: Kaprada (Rao, 2012), Dharampur, Nana Pondha (Vora, 1980)

*Hygrophila serpyllum T.Anderson [= Physichilus serpyllum Nees]

Herb; Fl. and Fr.: September - December

Anand Dt.: Khambhat (Bhagwanani, 1980); Arvalli Dt.: Modasa, Vatrak (Saxton and Sedgwick, 1918); Banaskantha Dt.: Hathidhara forest (Meena, 2012); Dahod Dt.: Devgadh Baria; Dang Dt. (Tadvi, 2013): Bheskatri, Galkund, Sunda (Yadav, 1979), Ahwa, Malegaon, Pipaldahad, Subir (Suryanarayana, 1968); Gir somnath Dt.; Junagadh Dt.; Kachchh Dt. (Patel et al., 2011); Narmada Dt.: Kelda, Namgir, Sankali, Waghumar (Pradeepkumar, 1993), Rajpipla (Shah, 1967); Navsari Dt.: Bansda (Desai, 1976); Panchmahal Dt.: Sankali; Porbandar Dt.: Barda (Bole and Pathak, 1988); Sabarkantha Dt.: Khedbrahma (Yogi, 1970; Bhatt, 1971; Bhatt and Sabnis, 1972; Parmar, 2012), Himmatnagar, Moti Posina, Vireshwar; Surat Dt.: Umarpada; Vadodara Dt.: Savli (Padate, 1973); Valsad Dt. (Inamdar and Patel, 1971): Pendha (Yadav, 1979), Parnera (More, 1972), Umbergaon (Bhagwanani, 1980)

*Justicia neesii Ramamoorthy [= Rostellularia neesii (Ramamoorthy) A.K.Mukh.]

Herb; Fl. and Fr.: September - December

Anand Dt.: Dhuvaran; Bharuch Dt.: Kavi; Dahod Dt.: Kanjeta; Junagadh Dt.: Girnar (Bole and Pathak, 1988); Navsari Dt.: Chari; Surat Dt.; Tapi Dt.: Gaumukh; Valsad Dt.: Pangarbari

*Justicia orbiculata T.Anderson

Herb; Fl. and Fr.: August - November

Ahmedabad Dt. (Saxton and Sedgwick, 1918; Yogi, 1970); Sabarkantha Dt.: Prantij (Saxton and Sedgwick, 1918; Parmar, 2012)

Neuracanthus sphaerostachys Dalzell

Shrub; Fl. and Fr.: August - October

Arvalli Dt.: Modasa (Yogi, 1970); Banaskantha Dt.: Danta on way to Hadad, Kangura-Umbrapani village near Ambaji (Meena, 2012), Vav; Bhavnagar Dt.: Mithivirdi (Meena, 2014b); Chhota udepur Dt.: Ambadungar, Pavi- 
jetpur, Satun (Desai, 2002), Kavant (Thaker, 1974); Dahod Dt.: Ratanmahal (Bedi, 1968), Devgadh Baria; Dang Dt. (Tadvi, 2013): Ghoghli, Subir (Yadav, 1979), Ahwa, Pipaldahad (Suryanarayana, 1968), Waghai; Devbhumi dwarka Dt.: Abhapar, Kileswar, Venu, Ghumli (Nagar, 2005); Gir somnath Dt. (Santapau and Raizada, 1954; Sisodia, 2007); Junagadh Dt.: Girnar (Bole and Pathak, 1988), Ramnath, Sapnes, Sasan (Menon, 1979), Jasadhar, Junvaniya, Tulsishyam (Bole and Pathak, 1988); Narmada Dt.: Kalvat, Shisha, Vav (Pradeepkumar, 1993), Rajpipla (Shah, 1967); Navsari Dt.: Bansda (Desai, 1976); Panchmahal Dt.: Pavagadh (Oza, 1961), Tuwa (Deshpande, 1968); Porbandar Dt.: Godhana, Satvirda, Adityana, Ranavav (Nagar, 2005); Sabarkantha Dt.: Khedbrahma (Bhatt, 1971), Mahudi, Pahada (Yogi, 1970), Dharod, Vadali (Parmar, 2012); Surat Dt.: Umarpada, Zankhvav (Yadav, 1979); Tapi Dt.: Vyara (Yadav, 1979); Vadodara Dt.: Savli (Padate, 1969; Padate, 1973); Valsad Dt. (Inamdar and Patel, 1971): Dighi (Rao, 2012), Dhamni, Sidhumbar (Yadav, 1979), Dharampur, Kaprada, Nana Pondha (Vora, 1980), Chival, Dhari, Parnera, Rabda, Udwada (More, 1972)

Neuracanthus tetragonostachyus subsp. trinervius (Wight) Bidgood [= Neuracanthus trinervius Wight]

Herb; Fl. and Fr.: November - March

Dang Dt. (Tadvi, 2013): Nilsakiya, Saputara (Yadav, 1979), Malegaon (Suryanarayana, 1968); Gir somnath Dt. (Sisodia, 2007); Valsad Dt.: Dharampur, Kaprada, Nanapondha (Vora, 1980), Huda, Tutarkhed

*Rungia elegans Dalzell and A.Gibson

Herb; Fl. and Fr.: September - January

Arvalli Dt.: Modasa; Bhavnagar Dt.: Palitana, Shatrunjaya (Meena, 2014b); Dang Dt. (Tadvi, 2013); Devbhumi dwarka Dt.: Beyt (Bole and Pathak, 1988); Jamnagar Dt.: Dhunvav, Ranjit Sagar, Rozi (Bole and Pathak, 1988); Rajkot Dt.: Pradhyuman park (Bole and Pathak, 1988)

Strobilanthes callosa Nees [= Carvia callosa (Nees) Bremek.]

Shrub; Fl. and Fr.: October - November

Saurashtra (Santapau, 1953; Bole and Pathak, 1988); Chhota udepur Dt.: Mogra, Kadipani, Turkheda, Kevdi, Jamlidam, Rangpur (Desai, 2002), Ambadungar (Thaker, 1974), Handev dungar, Kavant; Dahod Dt.: Kanjeta, Ratanmahal (Bedi, 1968); Dang Dt. (Tadvi, 2013): Malegaon, Saputara, Subir (Suryanarayana, 1968), Don, Galkund, Waghai; Narmada Dt.: Dabka, Fulsar, Namgir, Vaghumar (Pradeepkumar, 1993), Rajpipla (Shah, 1967), Gora; Navsari Dt.: Ambabari, Anklach, Mankunia, Vati (Desai, 1976); Panchmahal Dt.: Pavagadh (Oza, 1961); Sabarkantha Dt.: Himmatnagar; Valsad Dt.: Chavshala (Rao, 2012), Dharampur, Nana Pondha (Vora, 1980), Dhamni, Pendha

*Thelepaepale ixiocephala (Benth.) Bremek. [= Strobilanthes ixiocephala Benth.]

Shrub; Fl. and Fr.: November - January

Banaskantha Dt.: Ambaji (Meena, 2012); Dang Dt.; Junagadh Dt.: Girnar (Menon, 1979)

Bignoniaceae Juss:-

*Dolichandrone atrovirens (Roth) K.Schum. [= Bignonia atrovirens Roth; Dolichandrone crispa (Buch.-Ham. ex Roxb.) Seem.]

Tree; Fl. and Fr.: May - June

Dahod Dt.: Dumka, Garabdi (Bedi, 1968); Dang Dt.: Borkhal, Subir (Yadav, 1979); Valsad Dt.: Dharampur, Kaprada, Nana Pondha (Vora, 1980)

*Dolichandrone falcata (Wall. ex DC.) Seem. [= Dolichandrone lawii Seem.; Spathodea falcata Wall. ex DC.]

Tree; Fl. and Fr.: April - June

Ahmedabad Dt.; Chhota udepur Dt.: Bhadurpur, Boriyad, Ghantoli-songir, Koraj, Pani-mines, Sadhli, Vagasthal (Desai, 2002), Kavant (Thaker, 1974); Dang Dt. (Tadvi, 2013): Ahwa (Suryanarayana, 1968), Ambapada, Surkai; Jamnagar Dt. (Nagar, 2005); Kheda Dt.: Majham river (Yogi, 1970); Narmada Dt.: Gora, Gumandev, Netrang, (Patel, 1971), Fulsar, Junaraj, Pankhala (Pradeepkumar, 1993), Vankal (Yadav, 1979), Rajpipla (Shah, 1967); Navsari Dt.: Bansda; Panchmahal Dt.: Tuwa (Deshpande, 1968), Shivarajpur; Porbandar Dt. (Nagar, 2005); Sabarkantha Dt.: Khedbrahma (Bhatt, 1971; Bhatt and Sabnis, 1972), Dhansura (Saxton and Sedgwick, 1918), Meghraj (Parmar, 2012); Vadodara Dt.: Savli (Padate, 1969); Boriyad, Khakharia, Samlaya, Vejpur; Valsad Dt. (Inamdar and Patel, 1971): Dharampur, Kaprada, Nana Pondha (Vora, 1980), Parnera (More, 1972), Dungri (Patel RM, 1971), Kaprada (Rao, 2012)

*Heterophragma quadriloculare (Roxb.) K.Schum. [= Bignonia quadrilocularis Roxb.; Heterophragma roxburghii (Spreng.) DC.; Spathodea roxburghii Spreng.]

Tree; Fl. and Fr.: February - June

Ahmedabad Dt.; Dang Dt. (Tadvi, 2013): Bheskatri, Borkhal, Galkund, Mahal, Sagbara, Subir (Yadav, 1979), Ahwa, Malegaon, Pipaldahad (Suryanarayana, 1968), Kalibel; Devbhumi dwarka Dt.: Abhapara (Bole and Pathak, 1988); Narmada Dt.: Kalvat, Ninaighat, Thavadia (Pradeepkumar, 1993), Ghantoli (Yadav, 1979), Laldarwaja, 
Rajpardi (Patel, 1971), Fulsar, Kokam, Namgir, Sagai (Sharma, 2010); Navsari Dt.: Bansda; Surat Dt.: Umarpada (Yadav, 1979); Tapi Dt.: Kelvan, Rani Amba; Vadodara Dt.: MSU campus (Sabnis, 1967), Tarsali; Valsad Dt.: Lavkar (Rao, 2012), Chavshala, Avdha, Pendha (Yadav, 1979), Nana Pondha, Kaprada (Vora, 1980), Bhilad, Huda, Pangarbari

*Radermachera xylocarpa (Roxb.) K.Schum. [= Bignonia xylocarpa Roxb.]

Tree; Fl. and Fr.: April - September

Bharuch Dt.: Netrang; Chhota udepur Dt.: Kawant, Jamlidam (Desai, 2002), Ambadungar (Thaker, 1974), Hampheshwar, Kadipani, Turkheda; Dang Dt. (Tadvi, 2013): Waghai (Yadav, 1979); Narmada Dt.: Kokati, Shisha (Pradeepkumar, 1993), Mal, Samot (Joshi, 1983), Piplod (Sharma, 2010), Sagbara; Panchmahal Dt.: Jambughoda; Valsad Dt.: Dharampur, Kaprada, Nanapondha (Vora, 1980), Pangarbari

Lamiaceae Martinov:-

*Anisomeles heyneana Benth.

Herb; Fl. and Fr.: October - December

Chhota udepur Dt.: Kadipani-Hampheshwar road (Desai, 2002), Kavant (Thaker, 1974); Dahod Dt.: Alindra, Banvaro, Pipargota (Bedi, 1968); Dang Dt. (Tadvi, 2013): Ahwa, Malegaon, Pipaldahad, Subir (Suryanarayana, 1968), Mahal; Narmada Dt.: Sagbara, Ghatoli, Rajpipla; Panchmahal Dt.: Pavagadh (Oza, 1961); Tapi Dt.: Vyara; Valsad Dt.: Tukwada (Rao, 2012), Hedri (Yadav, 1979), Nana Pondha, Pangarbari (Vora, 1980), Tamachhadi

*Lavandula bipinnata (Roth) Kuntze [= Bystropogon bipinnatus Roth]

Undershrub; Fl. and Fr.: October - January

Bhavnagar Dt.: Palitana (Menon, 1979), Hastagiri (Meena, 2014b); Chhota udepur Dt.: Kavant (Thaker, 1974); Dang Dt. (Tadvi, 2013): Saputara (Yadav, 1979), Ahwa, Malegaon, Subir (Suryanarayana, 1968), Don; Devbhumi dwarka Dt.: Abhapara, Ghumli (Bole and Pathak, 1988), Kileswar, Venu (Nagar, 2005); Gir somnath Dt. (Santapau and Raizada, 1954); Jamnagar Dt. (Nagar, 2005); Junagadh Dt.: Girnar (Menon, 1979); Kachchh Dt. (Patel et al., 2011): Bhuj (Bhatt, 1993); Narmada Dt.: Chopdi (Pradeepkumar, 1993); Porbandar Dt.: Godhana, Satvirda (Thaker, 1910; Nagar, 2005)

*Nepeta bombaiensis Dalzell

Herb; Fl. and Fr.: July - August

Chhota udepur Dt.: Kavant; Junagadh Dt.: Girnar (Bole and Pathak, 1988); Panchmahal Dt.: Pavagadh (Oza, 1961)

*Pogostemon purpurascens Dalzell

Herb; Fl. and Fr.: October - January

Dang Dt. (Tadvi, 2013): Malegaon (Yadav, 1979), Mahal, Saputara, Subir (Suryanarayana, 1968); Narmada Dt.: Chopadi, Waghumar (Pradeepkumar, 1993), Dediapada; Sabarkantha Dt.: Khedbrahma (Bhatt and Sabnis, 1972); Valsad Dt.: Hedri (Yadav, 1979)

Lentibulariaceae Rich:-

*Utricularia graminifolia Vahl [= Utricularia purpurascens Graham]

Herb; Fl. and Fr.: September - October

Valsad Dt.: Dharampur, Mandva (Vora, 1980)

Oleaceae Hoffmanns. and Link:-

*Jasminum malabaricum Wight

Climbing shrub; Fl. and Fr.: March - May

Dahod Dt.: Kanjeta, Pipargota (Bedi, 1968); Dang Dt. (Tadvi, 2013); Navsari Dt.: Bansda (Desai, 1976), Vedchha; Valsad Dt.: Malegam, Rohina, Sanjan (Patel, 2013), Dharampur, Jogvel, Mandva, Nana Pondha (Vora, 1980), Abrama, Ghadoi, Marla (Patel RM, 1971), Sarigam, Tukwada (Rao, 2012), Rabda (More, 1972)

\section{Convolvulaceae Juss:-}

*Argyreia boseana Santapau and V.Patel

Climber; $F l$. and Fr.: July - December

Narmada Dt.: Dediapada (Patel, 2013)

*Argyreia sericea Dalzell

Climber; $F l$. and Fr.: August - October

Bhavnagar Dt.: Memon boarding (Meena, 2014b); Chhota udepur Dt.: Kavant (Thaker, 1974; Patel, 2013), Ambadungar (DNT1067, Thakar, 1970); Dahod Dt.: Devgadh Baria (Patel, 2013), Banvaro, Pipargota (Bedi, 1968); Dang Dt. (Tadvi, 2013): Ahwa (Yadav, 1979), Malegaon, Pipaldahad, Saputara, Subir (Suryanarayana, 1968); Gir 
somnath Dt. (Sisodia, 2007); Junagadh Dt.: Girnar, Junvaniya, Sasan (Bole and Pathak, 1988); Narmada Dt.: Dabka, Mathavali, Sagai (Pradeepkumar, 1993), Chanderpada (Yadav, 1979), Gora (Patel, 1971); Navsari Dt.: Bansda (Patel, 2013), Ambabari, Mahuvas, Manpur, Sara, Vati (Desai, 1976); Rajkot Dt.: Gondal (Bole and Pathak, 1988); Vadodara Dt.: Ajwa; Valsad Dt.: Malegam, Pindval (Patel, 2013), Avdha (Yadav, 1979), Dharampur, Kaprada, Nana Pondha (Vora, 1980), Paria (More, 1972), Saron (Patel RM, 1971), Tokarpada (Rao, 2012)

*Merremia rajasthanensis Bhandari

Climber; Fl. and Fr.: September - December

Kachchh Dt. (Joshi, 1994; Patel et al., 2011): Narayan sarovar (Patel, 2013), Mindhiyari (Parmar and Singh, 2003; Pandey et al., 2009)

*Merremia rhynchorrhiza Hallier f.

Climber; Fl. and Fr.: July - August

Bhavnagar Dt. (Menon, 1979; Meena, 2014b); Gir somnath Dt. (Bole and Pathak, 1988; Sisodia, 2007); Narmada

Dt.; Panchmahal Dt.; Valsad Dt.: Pendha (Yadav, 1979); Khatradevi, Umarpada (Patel, 2013)

Solanaceae Juss:-

Solanum hovei Dunal

Shrub; Fl. and Fr.: December - January

Ahmedabad Dt.: Dholka, near Sabarmati river

*Solanum purpureilineatum Sabnis and Bhatt

Herb; Fl. and Fr.: October - March

Anand Dt.: Khambhat (Bhagwanani, 1980); Banaskantha Dt.: Tharad; Chhota udepur Dt.: Hampheswar (Thaker, 1974), Kadipani, Kavant; Kachchh Dt.: Bhuj (KSR44, Rao, 1977; Bhatt, 1993); Sabarkantha Dt.: Khedbrahma (Bhatt and Sabnis, 1972), Dan Mahudi, Padhara (Bhatt, 1971; Parmar, 2012); Vadodara Dt. (Sabnis, 1967): Savli (Padate, 1973)

Apiaceae Lindl:-

*Heracleum grande (Dalzell and A.Gibson) Mukhop. [= Pastinaca grandis Dalzell and A. Gibson]

Herb; Fl. and Fr.: July - August

Dang Dt.: Malegaon, Saputara (Suryanarayana, 1968)

*Pimpinella adscendens Dalzell

Herb; Fl. and Fr.: December - April

Dang Dt.: Saputara; Kachchh Dt.: Dhinodhar, Nakhatrana (Bhatt, 1993); Narmada Dt.: Chopadi, Ninaighat (Pradeepkumar, 1993); Valsad Dt. (Inamdar and Patel, 1971): Vavar (Rao, 2012), Dharampur, Kolak, Tan, Man, Vanki (Vora, 1980), River Par (More, 1972), Atak, Ghadoi, Pardi (Patel RM, 1971)

*Pimpinella tomentosa Dalzell ex C.B.Clarke [= Heracleum tomentosum Dalzell and A.Gibson]

Herb; Fl. and Fr.: November - December

Dang Dt. (Tadvi, 2013): Gira-Giri Hills, Saputara (Suryanarayana, 1968); Valsad Dt.: Dharampur, Kaprada, Nanapondha (Vora, 1980)

*Pimpinella wallichiana (Miq.) Gandhi

Herb; Fl. and Fr.: September - December

Dang Dt. (Tadvi, 2013); Valsad Dt.: Rabda, River Auranga at Atak Pardi, Suliya (Rao, 2012)

Asteraceae Bercht. and J. Presl:-

*Artemisia nilagirica (C.B.Clarke) Pamp.

Herb; Fl. and Fr.: September - March

Banaskantha Dt.: Ambaji (Meena, 2012); Valsad Dt.: Dharampur, Kaprada, Nanapondha (Vora, 1980), Parnera (More, 1972)

*Phyllocephalum scabridum (DC.) K.Kirkman [= Decaneurum scabridum DC.; Baccharoides scabrida (DC.) M.R.Almeida; Centratherum molle (Wall. ex DC.) Benth. and Hook.f.; Decaneurum molle (Wall.) DC.]

Herb; Fl. and Fr.: September - October

Chhota udepur Dt.: Ambadungar, Kavant; Dahod Dt.: Alindra, Banvaro (Bedi, 1968); Dang Dt. (Tadvi, 2013):

Ahwa; Panchmahal Dt.: Pavagadh (Oza, 1961); Valsad Dt.: Dhamni, Kaprada

*Blumea belangeriana DC.

Herb; Fl. and Fr.: December - March 
Dang Dt.: Ahwa, Nilsakiya (Yadav, 1979), Malegaon, Saputara (Suryanarayana, 1968); Junagadh Dt.: Girnar (Menon, 1979); Narmada Dt.: Sagbara; Navsari: Bansda (Desai, 1976); Surat Dt.: Zankhvav (Yadav, 1979); Valsad Dt. (Patel RM, 1971): Kaprada (Rao, 2012), Dhamni, Hedri, Parnera, Penda (Yadav, 1979), Pangarbari

*Blumea eriantha DC.

Herb; Fl. and Fr.: October - December

Ahmedabad Dt.: Raypur (Saxton and Sedgwick, 1918), Viramgam (Meena, 2014a), Kharicut canal; Arvalli Dt.: Vatrak (Saxton and Sedgwick, 1918); Banaskantha Dt.: Mahudi (Yogi, 1970), Dantiwada, Palanpur (Patel, 2009); Bharuch Dt.; Bhavnagar Dt.: Shatrunjaya dam (Meena, 2014b); Chhota udepur Dt.: Kawant (Thaker, 1974), Gabadia, Kadipani, Hampeshwar, Satun, Ucheda, Udhwania, Zoz (Desai, 2002); Dahod Dt.: Alindra, Banvaro, Ratanmahal (Bedi, 1968); Dang Dt. (Tadvi, 2013): Bheskatri, Mahal (Yadav, 1979), Ahwa, Malegaon, Pipaldahad, Saputara, Subir (Suryanarayana, 1968); Gandhinagar Dt.: Mansa (Yogi, 1970); Junagadh Dt.: Girnar, Sasan (Menon, 1979); Narmada Dt.: Dumkhal, Sagai, Vav (Pradeepkumar, 1993), Umarkui, Umarpada (Yadav, 1979); Navsari Dt.: Bansda (Desai, 1976); Panchmahal Dt.: Pavagadh (Oza, 1961), Tuwa (Deshpande, 1968); Sabarkantha Dt.: Kotda, Khedbrahma, Isari, Fatehpur, Idar (Parmar, 2012); Vadodara Dt. (Sabnis, 1967): Savli (Padate, 1973), Chokari (Bhagwanani, 1980); Valsad Dt. (Inamdar and Patel, 1971; Patel RM, 1971; Vora, 1980): Chavshala, Nana Pondha, Pendha (Yadav, 1979), Pardi, Parnera (More, 1972), Kaprada (Rao, 2012)

Blumea malcolmii (C.B.Clarke) Hook.f. [= Pluchea malcolmii C.B.Clarke]

Herb; Fl. and Fr.: November - January

Chhota udepur Dt.: Rangpur (Thaker, 1974); Dang Dt.: Ahwa, Malegaon (Yadav, 1979), Saputara (Suryanarayana, 1968); Junagadh Dt. (Bole and Pathak, 1988); Navsari Dt.: Bansda (Desai, 1976); Panchmahal Dt.: Pavagadh (Oza, 1961); Porbandar Dt.: Satvirda, Adityana, Ranavav (Nagar, 2005); Surat Dt.; Surendranagar Dt.: Rangpur; Vadodara Dt.: Savli (Padate, 1973); Valsad Dt.: Rabda, Tutarkhed

*Goniocaulon indicum (Klein ex Willd.) C.B.Clarke [= Amberboa indica (Klein ex Willd.) DC.; Serratula indica Klein ex Willd.; Goniocaulon glabrum Cass.]

Herb; Fl. and Fr.: December - February

Banaskantha Dt.: Balaram-Ambaji wildlife sanctuary (Meena, 2012); Chhota udepur Dt.: Kavant (Thaker, 1974); Dang Dt. (Tadvi, 2013); Gir somnath Dt. (Sisodia, 2007): Veraval (Bole and Pathak, 1988); Junagadh Dt.: Sasan, Visavadar (Bole and Pathak, 1988); Narmada Dt.: Mokhadi, Surpan, Thavadia (Pradeepkumar, 1993); Navsari Dt.: Bansda, Lakawadi, Mahuvas, Sara, Pratapnagar (Desai, 1976); Sabarkantha Dt. (Parmar, 2012): Khedbrahma (Bhatt, 1971); Vadodara Dt.: Kamalpura, Pratapnagar

Helichrysum cutchicum (C.B.Clarke) R.S.Rao and Deshp.

Herb; Fl. and Fr.: August - September

Devbhumi dwarka Dt.: Abhapar, Beyt (Bole and Pathak, 1988), Okhamandal (Bole and Pathak, 1988); Jamnagar Dt.: Laloi, Kota (Bole and Pathak, 1988); Junagadh Dt.: Girnar; Kachchh Dt. (Rao and Deshpande 1968; Raole, 1993; Patel et al., 2011): Bhuj, Dahisara (Rao, 1981), Dhinodhar (Shah, 1978), Kotda, Lakhpat, Mosuna, Nakhatrana, Nani Khakhar (Rao, 2002), Hamankhudi, Narayan Sarovar (Pandey et al., 2009), Nadibaugh Rakhal, Naliya (Raole, 1993), Banni, Bharasar, Kala dungar, Mandvi, Mundra, Sheh (Joshi et al., 2013), Tapkeshwari (Joshi et al., 2012); Morbi Dt.: Ravapar (Rao, 2002); Porbandar Dt.: Barda (Shah, 1987)

*Pulicaria wightiana (DC.) C.B.Clarke [= Poloa wightiana DC.]

Herb; Fl. and Fr.: October - January

Banaskantha Dt.: Mahudi (Yogi, 1970), Dantiwada, Palanpur (Patel, 2009); Bhavnagar Dt.: Mahuva (Meena, 2014b); Dang Dt. (Tadvi, 2013): Subir (Suryanarayana, 1968); Devbhumi dwarka Dt.: Bhanvad, Kota (Bole and Pathak, 1988); Gandhinagar Dt.: Mansa (Yogi, 1970); Gir somnath Dt. (Santapau and Raizada, 1954): Somnath reclamation (Bole and Pathak, 1988); Jamnagar Dt.: Rozibet (Bole and Pathak, 1988); Junagadh Dt. (Gopal, 1983): Girnar, Khamba, Ramnath, Sapnes (Menon, 1979); Kachchh Dt. (Patel et al., 2011): Mindhiyari (Pandey et al., 2009); Kheda Dt.: Kapadvanj; Panchmahal Dt.: Pavagadh (Oza, 1961); Rajkot Dt. (Thakrar, 1987); Sabarkantha Dt.: Danmahudi (Bhatt, 1971), Himmatnagar, Idar (Yogi, 1970), Raigarh (Parmar, 2012), Prantij

Senecio bombayensis N.P.Balakr.

Herb; Fl. and Fr.: September - November

Dang Dt.: Malegaon, Saputara; Junagadh Dt.: Girnar; Panchmahal Dt.: Pavagadh (Oza, 1961)

*Senecio dalzellii C.B.Clarke [= Senecio lawii C.B.Clarke]

Herb; Fl. and Fr.: December - January

Panchmahal Dt.: Pavagadh (Oza, 1961)

*Tricholepis amplexicaulis C.B.Clarke

Herb; Fl. and Fr.: October - January 
Dang Dt. (Tadvi, 2013): Gira-Giri hills, Saputara (Suryanarayana, 1968); Devbhumi dwarka Dt.: Abhapara (Bole and Pathak, 1988), Kileswar, Venu (Nagar, 2005); Gir somnath Dt. (Sisodia, 2007); Junagadh Dt.: Gir

Tricholepis glaberrima DC.

Herb; Fl. and Fr.: December - January

Ahmedabad Dt. (Meena, 2014a); Anand Dt.: Khambhat (Bhagwanani, 1980); Banaskantha Dt.: Balaram-Ambaji wildlife sanctuary (Meena, 2012); Bharuch Dt. (Gopal, 1983); Chhota udepur Dt.: Ambadungar, Bhadurpur, Hampeshwar, Turkheda (Desai, 2002), Kavant (Thaker, 1974); Dahod Dt.: Ratanmahal (Bedi, 1968), Devgadh Baria; Dang Dt. (Tadvi, 2013): Ahwa, Malegaon, Saputara (Suryanarayana, 1968); Devbhumi dwarka Dt.: Bhanvad (Bole and Pathak, 1988), Abhapar, Kileswar, Venu, Ghumli (Nagar, 2005); Gir somnath Dt. (Santapau and Raizada, 1954); Jamnagar Dt.: Rozibet (Bole and Pathak, 1988); Junagadh Dt.: Girnar (Menon, 1979; Bole and Pathak, 1988), Sasan (Bole and Pathak, 1988); Mehsana Dt.: Visnagar (Bharati, 1959); Panchmahal Dt.: Pavagadh (Oza, 1961), Vishwamitri riverbank (Chavan and Sabnis, 1960); Porbandar Dt.: Godhana (Thaker, 1910; Nagar, 2005); Sabarkantha Dt.: Khedbrahma (Bhatt, 1971; Bhatt and Sabnis, 1972), Vireshwar; Vadodara Dt. (Sabnis, 1967): Savli (Padate, 1969; Padate, 1973), MSU campus (Phatak and Joshi, 1955); Valsad Dt.: Chavsari, Dhabhdi, Kaprada, Mandva (Vora, 1980), Umbergaon (Bhagwanani, 1980), Nana Pondha (Gopal, 1983)

\section{Discussion:-}

Among the total 192 Indian endemics found in Gujarat, dicotyledons dominate the endemic flora with 137 taxa (71\%) belonging to 97 genera under 33 families, whereas monocots are represented by 55 taxa (29\%) belonging to 37 genera under 9 families. The top five families of angiosperms, rich in endemic species contribute $57 \%$ of the total endemic species reported from Gujarat. Family Fabaceae has the largest number of Indian endemics (31 taxa), which is followed by Poaceae (27 taxa), Acanthaceae (20 taxa), Asteraceae (12 taxa) and Orchidaceae (10 taxa).

An analysis of the life-form types indicates that the endemic herbs $(66 \%)$ dominate the study area, followed by shrubs (12\%), trees (11\%), climbers $(6 \%)$, undershrubs $(3 \%)$ and climbing shrubs $(2 \%)$.

It was observed that large number of endemic herbs, climbers and shrubs flower in the months of SeptemberOctober; while the peak flowering for trees was observed in April (Fig. 2), which is the hottest season in Gujarat.

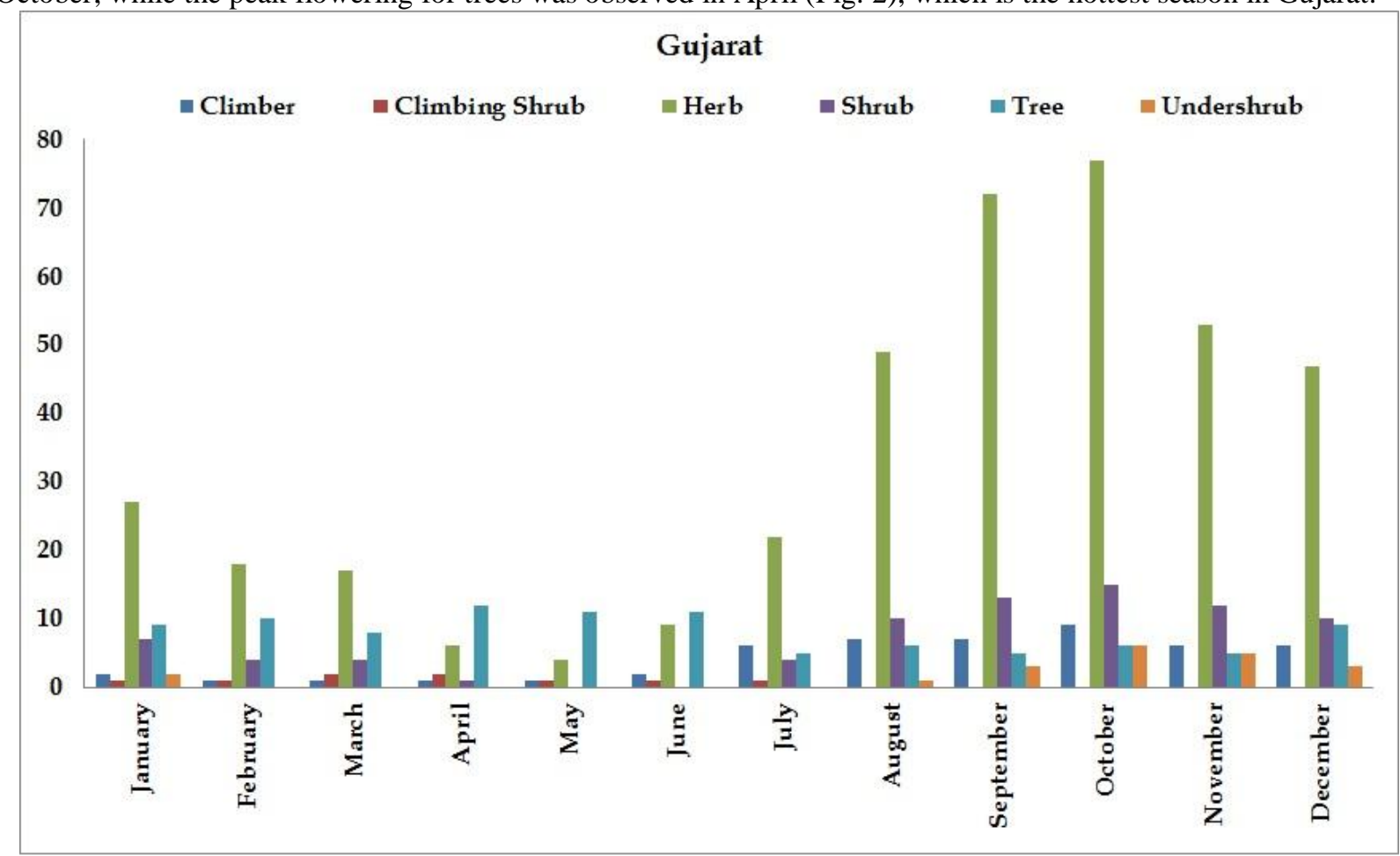

Figure 2-: Flowering phenology of endemic species in different life-forms

Analysis on the district wise distribution of endemic angiosperms shows that Dangs has maximum number of endemic angiosperms with 115 taxa, followed by Valsad (104), Narmada (71), Junagadh (63) and Panchmahal (57). 


\section{Kachchh:-}

The survey resulted in the documentation of 24 endemic taxa. Of which all of them are dicots belonging to 23 genera under 15 families, and no endemic monocots. Top 4 families contribute $54 \%$ of the total endemic species reported from Kachchh. Family Fabaceae has the largest number of endemics (6 taxa), which is followed by Apocynaceae (3 taxa), and Acanthaceae and Asteraceae (2 taxa each), remaining families with single taxa.

An analysis of the life-form types indicates that the endemic herbs (10) dominate the study area, followed by shrubs (6), trees (2), climbers (4) and undershrubs (2).

\section{Saurashtra:-}

The survey resulted in the documentation of 87 endemic taxa. Of which Dicots dominate with 70 taxa (80\%) belonging to 53 genera under 23 families, whereas monocots are represented by 17 taxa (20\%) belonging to 16 genera under 7 families. Top 5 families contribute $60 \%$ of the total endemic species reported from Saurashtra. Fabaceae family has the largest number of endemics (15), which is followed by Acanthaceae (13), Poaceae (10), Asteraceae (9) and Euphorbiaceae (5).

An analysis of the life-form types indicates that the endemic herbs (51) dominate the study area, followed by trees (14), shrubs (12), climbers (6) and undershrubs (4).

\section{Northern Gujarat:-}

The survey resulted in the documentation of 57 endemic taxa. Of which Dicots dominate with 46 taxa (81\%) belonging to 41 genera under 19 families, whereas monocots consists of 11 taxa (19\%) belonging to 10 genera under 5 families. Top 5 families contribute $63 \%$ of the total endemic species reported from Gujarat. Family Acanthaceae has the largest number of endemics (13), followed by Fabaceae (9), Poaceae (6), Asteraceae (5) and Apocynaceae (3).

An analysis of the life-form types indicates that the endemic herbs (33) dominate the study area, followed by shrubs (9), trees (9), climbers (3) and undershrubs (3).

\section{Central Gujarat:-}

The survey resulted in the documentation of 100 endemic taxa. Of which Dicots dominate with 76 taxa (76\%) belonging to 62 genera under 30 families, whereas monocots are represented by 24 taxa (24\%) belonging to 17 genera under 6 families. Top 5 families contribute $49 \%$ of the total endemic species reported from Gujarat. Family Fabaceae has the largest number of endemics (15), Acanthaceae (12), Poaceae (9), Asteraceae (8) and Orchidaceae (5).

An analysis of the life-form types indicates that the endemic herbs (60) dominate the study area, followed by trees (16), shrubs (9), climbers (8), undershrubs (4) and climbing shrubs (3).

\section{Southern Gujarat:-}

The survey resulted in the documentation of 157 endemic taxa. Of which Dicots dominate with 108 taxa (69\%) belonging to 74 genera under 28 families, whereas monocots represent 49 taxa (31\%) belonging to 33 genera under 8 families. Top 5 families contribute $54 \%$ of the total endemic species reported from Gujarat. Family Poaceae has the largest number of endemics (24), followed by Fabaceae (22), Acanthaceae (19), Asteraceae and Orchidaceae (10 taxa each).

An analysis of the life-form types indicates that the endemic herbs (95) dominate the study area, followed by trees (19), shrubs (17), climbers (10), undershrubs (4) and climbing shrubs (1).

The present study demonstrates that endemics are mostly concerted in southern Gujarat region, they are primarily herbaceous, and size of the family is directly proportional to the number of endemics. Moreover, many of the endemics are habitat specific, while some of them are known either only by type collections of just a few additional collections.

\section{Acknowledgments:-}

The study was made possible by a research grant provided by the Ruffords Small Grants (19988-1), and laptop donated by the IdeaWild Foundation. We are thankful to the support provided by Dr. S.L. Meena, Botanical Survey of India, Jodhpur and Dr. A.P. Singh (Chief Conservator of Forests, Gujarat) during extensive fieldwork in the study area. We would like to thank the Head, Department of Botany, The Maharaja Sayajirao University of Baroda for providing lab facilities. 


\section{References:-}

1. Anjaria, K.B. (2002). Floristic studies of Anand District. PhD Thesis. Sardar Patel University, Vallabh Vidyanagar, Gujarat.

2. Bahadur K.N. and Bennet S.S.R. (1978) A new species of Wrightia R.Br. (Apocynaceae) from India. Indian Forester: $32-35$.

3. Baxi, U.S. (2003) A taxonomic study of family Euphorbiaceae from Saurashtra. Ph.D. Thesis, Bhavnagar University, Bhavnagar.

4. Bedi, S.J. (1968). Floristic study of Ratan-mahal and surrounding hills, vols. 1 and 2. $\mathrm{PhD}$ thesis submitted to The Maharaja Sayajirao University of Baroda.

5. Bedi, S.J., Sabnis, S.D. and Bhatt, R.P. (1968). Additions to the Flora of Pavagadh hills, Gujarat State. J. Bombay Nat. Hist. Soc. 65(2): 522-525.

6. Bhagwanani P.P. (1980) Floristic and vegetational study of the Gujarat coast Khambhat to Umargam, vols. 1 and 2. Ph.D. Thesis. M.S. University, Baroda, Vadodara.

7. Bhambra, G.P. (2015) Phytochemical and pharmacological characterization of two endemic Tephrosia Pers. species from western India. Ph.D. Thesis. M.S. University, Baroda, Vadodara.

8. Bharati S.G. (1959) A brief account of the Flora of Visnagar, N. Gujarat and its environs. J. Bombay Nat. Hist. Soc. 56. 588-610.

9. Bhatt R.P. and Sabnis S.D. (1972) Further contribution to the flora and vegetation of Khedbrahma region of north Gujarat. Journ. M.S. Uni. Baroda: 7-34.

10. Bhatt, J.B. (1993). Studies on the flora of Western Kachh. Ph.D. Thesis, M.S. University of Baroda, Vadodara.

11. Bhatt, M.R. and Nagar, P.S. (2014). Habenaria foliosa A. Rich. (Orchidaceae) - A new record for the flora of Saurashtra, Gujarat. J. Econ. Taxon. Bot. Vol 38 (3-4): 552-554.

12. Bhatt, R.G. (1975) A contribution to the floristics and phytosociology of Panchmahals district in Gujarat state. Ph.D. Thesis, M.S. University of Baroda, Vadodara.

13. Bhatt, R.P. (1971). Studies on the flora and vegetation of Khedbrahma region in North gujarat with a note on the chromosome number and karyotype of some taxa therein, vols. 1, 2 and 3. Ph.D. Thesis, The Maharaja Sayajirao University of Baroda, Vadodara.

14. Binojkumar, M.S. (1993) A taxonomic revision of the genus Euphorbia L. (Euphorbiaceae) in India. Ph.D. Thesis, Bharathiar University.

15. Bole, P.V. and Pathak, J.M. (1988). Flora of Saurashtra, Part II and III, Botanical Survey of India, Calcutta.

16. Chavan, A.R. and Mehta, A.R. (1958a) Studies on the grasses of Gujarat-2, grasses of Baroda and its environs. Journ. M.S. Uni. Baroda 7(2): 67-83.

17. Chavan, A.R. and Mehta, A.R. (1958b) Grasses of Pavagarh. Journ. M.S. Uni. Baroda 7(2): 171-185.

18. Chavan, A.R. and Oza, G.M. (1960) Field notes on the systematics of Curcuma - Curcuma pseudomontana Graham at Pavagadh. Journal of the M. S. University Baroda 9(2): 41-42.

19. Chavan, A.R. and Oza, G.M. (1962). Contribution to the flora of Gujarat. Journal of the M. S. University Baroda 11(3): 79-80.

20. Chavan, A.R. and Oza, G.M. (1966). Contribution to the flora of the Dangs forest in Gujarat. Indian Forester. 92: 533-535.

21. Chavan, A.R. and Sabnis, S.D. (1958). Cyperaceae of Gujarat-1, the sedges of Baroda and its environs. Journ. M.S. Uni. Baroda 7(2): 57-66.

22. Chavan, A.R. and Sabnis, S.D. (1960). Along the banks of the river Vishwamitri. Indian Forester 86(8): 469474.

23. Chavan, A.R., Bedi S.J. and Sabnis, S.D. (1963) On useful plants of Devgadh hills, Gujarat state. Reprinted from Bulletin of the Botanical Society, College of Science, Nagpur 14(1): 25-32.

24. Cooke, T. (1958). The Flora of the presidency of Bombay. Reprinted BSI, Calcutta.

25. Desai, B.S. (2002) Floristic diversity and ethnobotany of Chhota-Udepur forest division. Ph.D. Thesis. M.S. University, Baroda, Vadodara.

26. Desai, M.J. (1976) A contribution to the flora of Bansda forests, vols. 1, 2 and 3. Ph.D. Thesis. Sardar Patel University, Vallabh Vidyanagar, Gujarat.

27. Desai, R.J. (2012). Studies on sedges and grasses of south Gujarat, vols. 1 and 2. Ph.D. Thesis. M.S. University, Baroda, Vadodara.

28. Deshpande, M.B. (1968). A contribution to the Floristic of Tuwa region, Panchmahals, vols. 1, 2 and 3. PhD Thesis. Sardar Patel University, Vallabh Vidyanagar, Gujarat.

29. eFloras (2017). Published on the Internet http://www.efloras.org [accessed 12 February 2017] Missouri Botanical Garden, St. Louis, MO \& Harvard University Herbaria, Cambridge, MA. 
30. Gopal, G.V. (1983) Ethnobotanical studies in the forest areas of some parts of Gujarat. Ph.D. Thesis. Sardar Patel University, Vallabh Vidyanagar, Gujarat.

31. Inamdar, J.A. (1968) A preliminary survey of the flora of Dharampur forests in Gujarat. Bull. Bot. Surv. India. 10: $126-132$.

32. Inamdar, J.A. and Patel, R.M. (1971). A preliminary Floristic survey of Bulsar- Tithal- Dungri areas in Gujarat State. Indian forester. 97(6): 322-331.

33. Jani, M.D. (2014) Bridging the knowledge gaps and developing an interactive system for identification of Gujarat flora. Ph.D. Thesis, Sardar Patel University, VallabhVidyanagar.

34. Joshi, J.V. (1980) A study of the flora of Surat and its environs. Ph.D. Thesis. M.S. University, Baroda.

35. Joshi, K.I. (1994) Floristic, phytosociological Cambay taluka and ethnobotanical study of Cambay taluka. Ph.D. Thesis, Sardar Patel University, VallabhVidyanagar.

36. Joshi, M.C. (1983) A floristic and phytochemical survey of some important south Gujarat Forests with special reference to plants of medicinal and ethnobotanical interest. Ph.D. Thesis, M.S. University, Baroda.

37. Joshi, P.N., H.B. Soni, Sunderraj S.F.W. and Joshua J. (2013) Status and distribution of threatened and endemic Helichrysum cutchicum (Asteraceae) in Arid Ecosystem of Kachchh, Gujarat, India. International Journal of Life Sciences Leaflets. 11 (11): 93-100.

38. Joshi, P.N., Joshi, E.B. and Jain B.K. (2012). Ecology and conservation of threatened plants in Tapkeshwari Hill ranges in the Kachchh Island, Gujarat, India. Journal of Threatened Taxa 4(2): 2390-2397.

39. Karetala, Y.Y. (1973). A contribution to the Floristic and Phytosociology of Chhota-Udepur Forests Division. $\mathrm{PhD}$ thesis submitted to Sardar Patel University, Vallabh Vidyanagar, Gujarat.

40. Malhotra, S.K. and Wadhwa, B.M. (1973). Studies on the Botany of Jamnagar District. M.V.K. Patrika 8(2): 323.

41. Mathew, T. and Nayar, M.P. (1989) A new species of Ammannia L. (Lythraceae) from India. Bull. Bot. Surv. India. 31(1-4): 158-161.

42. Meena, S.L. (2012) A checklist of the vascular plants of Banaskantha district, Gujarat, India. Nelumbo 54: 3991.

43. Meena, S.L. (2014a) An enumeration of the plants of Ahmedabad and Gandhinagar district of Gujarat, India. Nelumbo 56: 124-182.

44. Meena, S.L. (2014b) Floral diversity of Bhavnagar district, Gujarat state (India) - A checklist. Nelumbo 56: 183-224.

45. Meena, S.L. and Krishna P.H. (2015) A new generic record to the flora of Gujarat state (India): Hetrostemma dalzellii Hook.f. (Asclepiadaceae). J. Econ. Taxon. Bot. Vol. 39 No. 2 305-308.

46. Menon, A.R.R. (1979) Floristics and phytosociological studies of some parts of Saurashtra. Ph.D. Thesis, S.P. University, Vallabh Vidyanagar.

47. More, P.G. (1972). A contribution to the Flora of Parnera hills, Pardi and Udhwada area in South Gujarat. PhD thesis submitted to Sardar Patel University, Vallabh Vidyanagar, Gujarat.

48. Nagar P.S., Sata S.J., and Pathak S.J. (2003) Rediscovery of Tephrosia jamnagarensis (Fabaceae), An endangered and narrow endemic plant species of Saurashtra, Sida 20(4):1701-1705.

49. Nagar, P.S. (2005) Floristic biodiversity of Barda hills and its surroundings. Scientific Publisher, Jodhpur.

50. Nagar, P.S. (2007) A little known plant species of Gujarat Tephrosia collina var lanuginocarpa Sharma. J. Bombay Nat. Hist. Soc. (3):104.

51. Nagar, P.S. (2008) Medicinal Plants of Saurashtra, Gujarat. Bishen Singh Mahendra Pal Singh. Dehradun, India.

52. Oza, A.R. (1991) Taxonomical and ecological studies of the flora of and around Bhavnagar. Ph.D. Thesis. Saurashtra University, Rajkot.

53. Oza, G.M. (1961) Flora of Pavagadh, vols. 1 and 2. Ph.D. Thesis. M.S. University, Baroda, Vadodara.

54. Padate, S.N. (1969). A contribution to the flora of Savli taluka, Gujarat state, India. Reprinted from the Journal of the M. S. University Baroda 17(3) and 18(3): 101-112.

55. Padate, S.N. (1973). Studies on the flora and vegetation of Savli Taluka. Ph.D. Thesis. The M. S. University of Baroda.

56. Pandey R.P., Singh V. and Parmar P.J. (2009) Phytodiversity of the Narayan sarovar wildlife sanctuary, Gujarat, India. Nelumbo. 51: 41-98.

57. Parabia M.H. (1974) A contribution to the Cyperaceae of Gujarat State (Floristics, trichomes, glume anatomy and palynology), Part I and II. Ph.D. Thesis, S.P. University, Vallabh Vidyanagar.

58. Parmar, P.J. (2012) A checklist of the vascular plants of Sabarkantha district, Gujarat, India. Nelumbo 54: 92137. 
59. Parmar, P.J. and Singh, V. (2003) Interesting plant records from Gujarat, India. Ind. J. Forestry. 26(4): $418-420$.

60. Patel R., Patel Y., Kumar A., Mahato R. and Kumar V. (2014) Some observations on the distribution and new host plant for Corallocarpus conocarpus in Gujarat, India. International journal of plant, animal and environmental sciences. 4(1): 134-135.

61. Patel R.M. (1971) Flora of Bulsar and environs, Gujarat, Part I and II. Ph.D. Thesis, S.P. University, Vallabh Vidyanagar.

62. Patel Y.S., Patel R.M., Joshi P.N. and Dabgar Y.B. (2011) Study of angiospermic flora of Kachchh district, Gujarat, India. International Journal of Life Sciences Leaflets. 19: 739-768.

63. Patel, M.K. (2009) Study of floristic and ethnobotanical aspect of angiosperms of palanpur and dantiwada talukas of Banaskantha district, Gujarat. Ph.D. Thesis, Bhavnagar University, Bhavnagar.

64. Patel, R.I. (1965). Grasses of Gujarat State. Indian forester. 91(5): 309-340.

65. Patel, R.I. (1971). Forest Flora of Gujarat State. Forest Dept. Gujarat State. Baroda.

66. Patel, S.L. (2013) A botanical investigation on climbing plants of Gujarat. Ph.D. Thesis, S.P. University, Vallabh Vidyanagar.

67. Patil, S.N. and Sabnis, S.D. (1982). New Plant Records from Urban Evironment of Baroda, Gujarat. J. Bombay Nat. Hist. Soc. 79(1): 117-119.

68. Phatak, V.G. and Joshi, B.B. (1955) A brief account of the flora of the university campus, Baroda. Journal of the M. S. University Baroda: 125-158.

69. Phatak, V.G. and Oza, G.M. (1959). Occurrence of Curucma inodora Blatt. at Pavagadh (Gujarat). J. Bombay Nat. Hist. Soc. 56(2): 368-369.

70. Pradeepkumar, G. (1993). Vegetational and Ecological studies of Shoolpaneshwar WLS. Ph.D. Thesis, The Maharaja Sayajirao University of Baroda, Vadodara.

71. Punjani, B.L., Desai, P. and Patel, S.K. (2015) Some ethnobotanical plants used by the tribals of Jessore wildlife sanctuary area, Banaskantha district, Gujarat, India. Phytodiversity. 2(1): 39-44.

72. Raghavan, R.S., Wadhwa, B.M., Ansari, M.Y. and Rao, R.S. (1981). A Checklist of the plants of Gujarat. Records of Botanical Survey of India. 21: 1-120.

73. Rajendrakumar, S. and Kalavathy, S. (2010). Status and distribution of the 'rare' (r) tree species, Anogeissus sericea brandis var. nummularia king ex Duthie. in the forest of North Gujarat region (NGR),Gujarat, India. International Journal of Life Sciences Leaflets. 6:178 - 181.

74. Rao, K.S.S. (1981) Flora of South-eastern Kachchh. Ph.D. Thesis, M.S. University of Baroda, Vadodara.

75. Rao, R.S. and Deshpande, U.R. (1968) Helichrysum cutchicum (C.B. Cl.) R. Rao et Desh. - An interesting species from Western India. Bull. Bot. Surv. India. 10(2): 225-227.

76. Rao, V.H. (2012) A floristic and ethnobotanical survey of Kaprada (hilly forest) and Umbergaon (coastal) talukas of Valsad district. Ph.D. Thesis, Veer Narmad South Gujarat University, Surat.

77. Rao, V.R. (2002). Distributional Status survey of Threatened Plants of Gujarat. Ph.D. Thesis, S.P. University, Vallabh Vidyanagar.

78. Raole V.M., Desai R.J. and Veldkamp, J.F. (2011) Ischaemum sayajiraoi, a new species of Poaceae from Gujarat, India. Kew Bulletin, 66: 303-306.

79. Raole, V.M. (1993) Studies on Endangered and Endemic Desert Taxa - Kachchh. Ph.D. Thesis, M.S. University of Baroda, Vadodara.

80. Reddy, A.S. (1987) Flora of Dharampur forest, Part I and II. Ph.D. Thesis, S.P. University, Vallabh Vidyanagar.

81. Sabnis, S.D. (1962) Cyperaceae of Gujarat. Bull. Bot. Surv. India. 4(1-4): 193-197.

82. Sabnis, S.D. (1967) A study of the flora and vegetation of Baroda and environs including an account of the Cyperaceae of Gujarat. Ph.D. Thesis, The Maharaja Sayajirao University of Baroda, Vadodara.

83. Sabnis, S.D. and Bedi S.J. (1971) Ceropegia odorata Hook.f. (Asclepiadaceae): A little-known plant of western India. Kew Bulletin 25(1): 57-59.

84. Sanjappa, M. (1977) Cytotaxonomical studies in some genera of the tribes Indigofereae and Desmodieae of Fabaceae. Ph.D. Thesis, The Maharaja Sayajirao University of Baroda, Vadodara.

85. Sanjappa, M. (1992) Legumes of India. Bishen Singh Mahendra Pal Singh. Dehradun, India.

86. Santapau, H. (1953) Plants of Saurashtra - a preliminary list. Saurashtra Research Society, Rajkot.

87. Santapau, H. (1962) The Flora of Saurashtra Part-I. Ranunculaceae to Rubiaceae 1-270. Rajkot, 1-viii.pp.1270.

88. Santapau, H. and Kapadia, Z. (1966) The Orchids of Bombay. Government of India Publication, Calcutta.

89. Santapau, H. and Raizada, M.B. (1954) Contribution to the flora of the Gir forest in Saurashtra. 80(7): 379-389. 
90. Satish K.V., Pasha S.V., Hari Krishna P. and Reddy C.S. (2015) Achyranthes coynei Santapau (Amaranthaceae): An endemic and threatened species from Kachchh desert, India. National Academy Science Letters. 38(3).

91. Saxton, W.T. and Sedgwick, L.G. (1918). Plants of Northern Gujarat. Rec. Bot. Surv. India. 6: $207-323$.

92. Shah G.L. and Patel A.I. (1971). Some noteworthy plants of Gujarat. Indian Forester 97(11): 636-637.

93. Shah G.L. and Suryanarayana B. (1968) On the occurrence of Eriolaena stocksii Hk. f. and Th. from Gujarat. Indian Forester: 894-896.

94. Shah G.L. and Yogi D.V. (1974) Additions to the flora of North Gujarat (Ahmedabad-Mehsana-Sabarkantha districts). J. Bombay Nat. Hist. Soc. 71(1): 58-63.

95. Shah, G.L. (1967) A preliminary contribution to the flora of Rajpipla forests in Gujarat state. Indian Forester: 672-676.

96. Shah, G.L. (1978). Flora of Gujarat State, Part I and II. Sardar Patel University Press, Vallabh Vidyanagar.

97. Shah, G.L. (1983). Rare species with restricted distribution in South Gujarat. In: S.K.Jain and R.R.Rao. (eds.) An Assessment of Threatened Plants of India. Bot. Surv. India, Calcutta. p. 50-54.

98. Sharma, A.K. (2010) Studies on the diversity, distribution and local utility of medicinal plants in shoolpaneshwar wildlife sanctuary, Gujarat. Ph.D. Thesis, S.P. University, Vallabh Vidyanagar.

99. Shetty, B.V. and Pandey, R.P. (1992) A new species of Tamarix (Tamaricaceae) from Gujarat, India. Bull. Bot. Surv. India. 31(1-4): 152-153.

100.Singh P., Karthigeyan K., Lakshminarasimhan P. and Dash S.S. (2015) Endemic Vascular Plants of India, Botanical Survey of India, Kolkata: 355.

101. Singhadiya, M.K., Padhye, P.M. and Pandey, R.P. (2011) Addition to the flora of Gujarat state - India. J. Econ. Taxon. Bot. 35(1): 85-89.

102. Sisodia, P.S. (2007) Plant diversity and some ecological studies in Gir national park and sanctuary, vols. 1 and 2. Ph.D. Thesis. M.S. University, Baroda, Vadodara.

103. Suryanarayana, B. (1968) A contribution to the flora of Dangs forest, Gujarat, Part I, II, and III. PhD thesis submitted to Sardar Patel University, Vallabh Vidyanagar, Gujarat.

104. Tadvi, D.S. (2013) Floristic diversity of Dangs. Ph.D. Thesis. M.S. University, Baroda, Vadodara.

105.Thaker, D.N. (1974) Floristic and ethnobotanical studies on Kawant range forests in Central Gujarat. PhD thesis submitted to South Gujarat University, Surat.

106.Thaker, J.I. (1910) Vanaspati Sastara- Barda Dungarni Jadibuti tani Pariksha anae Upyog. (BOTANY- A complete and Comprehensive Account of the Flora of Barda Mountain (Kathiawad). Gujarati Printing Press. Bombay. p. 717. (2nd revised edition; edi. B. G. Shah. 1952: Sastu Sahitya Vardhak Karyalaya, Ahmedabad. p. 733.

107.Thakrar, N.K. (1987). Biological flora of Rajkot. Ph.D. Thesis, Saurashta University, Rajkot.

108. Vora, H.M. (1980). A contribution to the Flora of Dharampur, Kaprada and Nana Pondha ranges. PhD thesis submitted to Sardar Patel University, Vallabh Vidyanagar, Gujarat.

109. Vyas, K.J. (1973). A contribution to the Floristics and phytosociology of the river Narmada in Gujarat state. $\mathrm{PhD}$ thesis submitted to Sardar Patel University, Vallabh Vidyanagar, Gujarat.

110. Yadav, S.R. (1979). A contribution to the Flora of some parts of South Gujarat. PhD thesis submitted to Sardar Patel University, Vallabh Vidyanagar, Gujarat.

111. Yogi, D.V. (1970). A contribution to the Flora of North Gujarat. PhD thesis submitted to Sardar Patel University, Vallabh Vidyanagar, Gujarat. 